TRANSACTIONS OF THE

AMERICAN MATHEMATICAL SOCIETY

Volume 349, Number 8, August 1997, Pages 3149-3179

S $0002-9947(97) 01859-X$

\title{
AN INDEX FORMULA FOR ELLIPTIC SYSTEMS IN THE PLANE
}

\author{
B. ROWLEY
}

\begin{abstract}
An index formula is proved for elliptic systems of P.D.E.'s with boundary values in a simply connected region $\Omega$ in the plane. Let $\mathcal{A}$ denote the elliptic operator and $\mathcal{B}$ the boundary operator. In an earlier paper by the author, the algebraic condition for the Fredholm property, i.e. the Lopatinskii condition, was reformulated as follows. On the boundary, a square matrix function $\Delta_{\mathcal{B}}^{+}$defined on the unit cotangent bundle of $\partial \Omega$ was constructed from the principal symbols of the coefficients of the boundary operator and a spectral pair for the family of matrix polynomials associated with the principal symbol of the elliptic operator. The Lopatinskii condition is equivalent to the condition that the function $\Delta_{\mathcal{B}}^{+}$have invertible values. In the present paper, the index of $(\mathcal{A}, \mathcal{B})$ is expressed in terms of the winding number of the determinant of $\Delta_{\mathcal{B}}^{+}$.
\end{abstract}

\section{INTRODUCTION}

To indicate the idea of the proof of the index formula, let us consider first a special case. The region $\Omega \subset \mathbb{R}^{2}$ is assumed to be bounded with $C^{\infty}$ boundary, and simply connected. Consider the $2 \times 2$ system

$$
\begin{gathered}
\frac{\partial u}{\partial x_{2}}-\left(\begin{array}{cc}
0 & -1 \\
1 & 0
\end{array}\right) \frac{\partial u}{\partial x_{1}}+A_{0}(x) \cdot u=f(x), \quad x \in \Omega, \\
u_{1} \cdot \cos \varphi(y)-u_{2} \cdot \sin \varphi(y)=g(y), \quad y \in \partial \Omega,
\end{gathered}
$$

where $u=\left[\begin{array}{ll}u_{1} & u_{2}\end{array}\right]^{T}$ is a real 2-vector function. Writing this problem in complex form with $w=u_{1}+i u_{2}, z=x_{1}+i x_{2}$, and $\partial / \partial \bar{z}=\frac{1}{2}\left(\partial / \partial x_{1}+i \partial / \partial x_{2}\right)$, we obtain the Riemann-Hilbert problem:

$$
\begin{gathered}
\partial w / \partial \bar{z}+a(z) w+b(z) \bar{w}=f(z), \quad z \in \Omega, \\
\operatorname{Re} e^{i \varphi(y)} w=g(y), \quad y \in \partial \Omega,
\end{gathered}
$$

where $a, b \in C^{\infty}(\bar{\Omega})$ and $\varphi \in C^{\infty}(\partial \Omega)$. The $\mathbb{R}$-linear operator defined by $w \mapsto$ $\left(\partial w / \partial \bar{z}-a w-b \bar{w}, \operatorname{Re} e^{i \varphi} w_{\mid \partial \Omega}\right)$ is Fredholm in appropriate Sobolev spaces, its

Received by the editors August 16, 1994.

1991 Mathematics Subject Classification. Primary 35J40, 35J55, 15A22.

Key words and phrases. Elliptic boundary value problems, matrix polynomials, index formula, Riemann-Hilbert problem.

(C) 1997 American Mathematical Society 
solvability properties depend on the winding number, $\chi=\frac{1}{2 \pi}[\varphi(y)]_{\partial \Omega}$, and the index formula

$$
\text { index }=1-2 \chi=1-\frac{1}{\pi}[\varphi(y)]_{\partial \Omega}
$$

is well known; see, for instance, [We] or [Ga].

A Fredholm operator is a bounded linear operator whose kernel has finite dimension, $\alpha$, and whose image has finite codimension, $\beta$; the index of this operator is defined to be $\alpha-\beta$. The reader of this paper is assumed to know that the Lopatinskii condition (abbreviated from now as L-condition) is the necessary and sufficient condition for an elliptic system $(\mathcal{A}, \mathcal{B})$ to be Fredholm (in suitable Sobolev spaces); for instance, see [WRL, Chap. 9]. Assuming that this has been established, the proof of the index formula is a matter of dealing with the L-condition which is algebraic-topological in nature.

The proof of the index formula in the present paper uses the theory of matrix polynomials due to Gohberg, Lancaster, Rodman and others; see [GLR], [BGR]. The relevant parts of this theory which are needed here are developed in [Ro].

The structure of the proof of the index formula is as follows. Given a first-order elliptic system in the plane with coefficients that are matrices with real entries, one can transform it to a "generalized Riemann-Hilbert problem" by means of a similarity transformation in the dependent variable, and then construct a homotopy from this new problem to a diagonal system of Riemann-Hilbert problems to which the above formula may be applied; this is done in $\S 3$ and $\S 4$. Higher-order systems $(\mathcal{A}, \mathcal{B})$ are then handled in $\S 5$ by a standard device which involves a reformulation as a first-order system. Up to that point the index formula holds for systems with real matrix coefficients, but in $\S 6, \S 7$ we show how this restriction can be removed. In each case the proof of the index formula amounts to showing that the index of $(\mathcal{A}, \mathcal{B})$ is equal to the topological index defined in $\S 2$.

An index formula for higher-order elliptic systems in the plane was first proved by $\mathrm{Vol}^{\prime}$ pert [Vo] in 1961 for systems with real matrix coefficients. It is probably fair to say, however, that both the statement and proof of the formula are simpler in the present paper. Furthermore, there is no restriction here on the orders of the rows of the boundary operator as there was in [Vo].

A formula for the index of an elliptic system $(\mathcal{A}, \mathcal{B})$ on a compact manifold with boundary was found by Atiyah and Bott $[\mathrm{AB}]$ in 1964. By means of some topological arguments, they were able to reduce the index problem for $(\mathcal{A}, \mathcal{B})$ to the case of the index formula on compact, closed manifolds in [AS]. Applications of the theory of matrix polynomials to boundary value problems in higher dimensions are treated elsewhere, in [WRL].

\section{Elliptic Systems in the Plane: SOME PRELIMINARIES}

This section is a continuation of [Ro, $\S 3, \S 6, \S 7]$; the Lopatinskii condition is examined in further detail for differential operators in the plane.

By a region we mean a nonempty connected open set in the complex plane. Let $\Omega \subset \mathbb{R}^{2}$ be a bounded region with $C^{\infty}$ boundary. We denote by Ell ${ }^{\ell}$ the set of properly elliptic differential operators

$$
\mathcal{A}(x, \partial)=\sum_{|\alpha| \leq \ell} A_{\alpha}(x) \partial^{\alpha}, \quad x=\left(x_{1}, x_{2}\right) \in \bar{\Omega},
$$


with principal part, $\pi \mathcal{A}$, homogeneous of degree $\ell$. The coefficients $A_{\alpha}$ are assumed to be smooth $p \times p$ matrix functions. Here we are using the usual multi-index notation: $\partial^{\alpha}=\partial_{1}^{\alpha_{1}} \partial_{2}^{\alpha_{2}},|\alpha|=\alpha_{1}+\alpha_{2}$, where $\partial_{j}=\partial / \partial x_{j}, j=1,2$. In a slight change of notation from [Ro, §6], we let $\mathrm{BE}^{\ell, \mathbf{m}}$ denote the set of all boundary problems $(\mathcal{A}, \mathcal{B})$ where $\mathcal{A} \in \mathrm{Ell}^{\ell}$ and $\mathcal{B}$ is a differential boundary operator of the form

$$
\mathcal{B}(y, \partial)=\left[\sum_{|\alpha| \leq m_{k}} b_{k}(y) \partial^{\alpha}\right]_{k=1}^{r}, \quad y \in \partial \Omega,
$$

satisfying the L-condition relative to $\mathcal{A}$. More general (pseudo-differential) boundary operators were permitted in [Ro] for the purpose of constructing homotopies of elliptic boundary problems, but for systems in the plane this turns out to be unnecessary (see Theorem 1.2).

Let $n(y)$ be the inward-pointing unit normal vector at $y \in \partial \Omega$, and $\tau(y)$ the unit tangent vector to $\partial \Omega$ such that $\tau(y), n(y)$ is a positively oriented basis of $\mathbb{R}^{2}$. We identify the unit cotangent bundle, $\operatorname{ST}^{*}(\partial \Omega)$, with the unit tangent bundle, $\mathrm{ST}(\partial \Omega)$. If $\left(y, \xi^{\prime}\right) \in \mathrm{ST}^{*}(\partial \Omega)$ then

$$
\left|\xi^{\prime}\right|=1, \quad \text { so } \quad \xi^{\prime}= \pm \tau(y),
$$

and $\mathrm{ST}^{*}(\partial \Omega)$ is the disjoint union of two copies of $\partial \Omega$.

Let $(\mathcal{A}, \mathcal{B}) \in \mathrm{BE}^{\ell, \mathbf{m}}$, i.e. $\mathcal{A}$ is an elliptic operator of the form (1a) and $\mathcal{B}$ is a boundary operator (1b) having order $m_{k}$ in the $k$ th row, $k=1, \ldots, r$. Associated with the principal part of $\mathcal{A}$ on the boundary $\partial \Omega$, there is the $p \times p$ matrix polynomial

$$
L_{y, \xi^{\prime}}(\lambda):=\pi \mathcal{A}\left(y, \xi^{\prime}+\lambda \cdot n(y)\right)=\sum_{j=0}^{\ell} A_{j}\left(y, \xi^{\prime}\right) \lambda^{j},
$$

$y \in \partial \Omega, 0 \neq \xi^{\prime} \in \mathrm{T}_{y}^{*}(\partial \Omega)$. (See $[\mathrm{Ro}, \S 3]$.) Now let $\tilde{L}_{y}(\lambda)=L_{y, \tau(y)}(\lambda)$, that is,

$$
\tilde{L}_{y}(\lambda)=\pi \mathcal{A}(y, \tau(y)+\lambda n(y))=\sum_{j=0}^{\ell} \tilde{A}_{j}(y) \lambda^{j}, \quad y \in \partial \Omega,
$$

where $\tilde{A}_{j}(y)=A_{j}(y, \tau(y))$. If $\left(\tilde{X}_{ \pm}(y), \tilde{T}_{ \pm}(y), \tilde{Y}_{ \pm}(y)\right)$ is a $\gamma^{ \pm}$-spectral triple of the matrix polynomial $\tilde{L}_{y}(\lambda)$ then we may define a $\gamma^{+}$-spectral triple of $L_{y, \xi^{\prime}}(\lambda)$ as follows:

$$
\left(X_{+}\left(y, \xi^{\prime}\right), T_{+}\left(y, \xi^{\prime}\right), Y_{+}\left(y, \xi^{\prime}\right)\right)= \begin{cases}\left(\tilde{X}_{+}(y), \tilde{T}_{+}(y), \tilde{Y}_{+}(y)\right), & \xi^{\prime}=\tau(y), \\ \left(\tilde{X}_{-}(y),-\tilde{T}_{-}(y),(-1)^{1-\ell} \tilde{Y}_{-}(y)\right), & \xi^{\prime}=-\tau(y) .\end{cases}
$$

We also define $\left(X_{-}\left(y, \xi^{\prime}\right), T_{-}\left(y, \xi^{\prime}\right), Y_{-}\left(y, \xi^{\prime}\right)\right)$ by the equation [Ro, (5-8)]. Associated with the boundary operator $\mathcal{B}$ there is the matrix polynomial

$$
\tilde{B}_{y}(\lambda)=\pi \mathcal{B}(y, \tau(y)+\lambda n(y))=\sum_{j=0}^{\mu} \tilde{B}_{j}(y) \lambda^{j}, \quad y \in \partial \Omega,
$$

where $\tilde{B}_{j}(y)=B_{j}(y, \tau(y))$ and $\mu \leq \max m_{k}$, and we let

$$
\tilde{\Delta}_{\mathcal{B}}^{ \pm}(y)=\sum_{j=0}^{\mu} \tilde{B}_{j}(y) \tilde{X}_{ \pm}(y) \tilde{T}_{ \pm}^{j}(y)
$$


and also

$$
\Delta_{\mathcal{B}}^{+}\left(y, \xi^{\prime}\right)=\sum_{j=0}^{\mu} B_{j}\left(y, \xi^{\prime}\right) X_{+}\left(y, \xi^{\prime}\right) T_{+}^{j}\left(y, \xi^{\prime}\right) .
$$

Note the following relationship between the matrix functions (4) and (5):

$$
\Delta_{\mathcal{B}}^{+}(y, \tau(y))=\tilde{\Delta}_{\mathcal{B}}^{+}(y), \quad \Delta_{\mathcal{B}}^{+}(y,-\tau(y))=M(-1) \tilde{\Delta}_{\mathcal{B}}^{-}(y),
$$

the second equality being valid for a differential operator $\mathcal{B}$, because $\pi \mathcal{B}(y, c \xi)=$ $M(c) \cdot \pi \mathcal{B}(y, \xi)$ for all $c \in \mathbb{C}$, in particular when $c=-1$. Here $M(c)$ is the $r \times r$ diagonal matrix $\left[c^{m_{k}} \delta_{k i}\right]$. In view of $[$ Ro, Theorem $3.3(\mathrm{iii})],(\mathcal{A}, \mathcal{B})$ satisfies the L-condition if and only if $\operatorname{det} \Delta_{\mathcal{B}}^{+}\left(y, \xi^{\prime}\right) \neq 0$ when $\xi^{\prime}= \pm \tau(y)$, which we now see is equivalent to the condition

$$
\operatorname{det} \tilde{\Delta}_{\mathcal{B}}^{ \pm}(y) \neq 0 \quad \text { for all } y \in \partial \Omega \text {. }
$$

Remark 1.1. If the coefficients of $\tilde{L}_{y}(\lambda)$ are real matrices then we may assume that $\left(\tilde{X}_{-}(y), \tilde{T}_{-}(y)\right)=\left(\tilde{X}_{+}(y), \overline{T_{+}(y)}\right)$. Further, if the coefficients of $\tilde{B}_{y}(\lambda)$ are also real matrices then we have $\tilde{\Delta}_{\mathcal{B}}^{-}(y)=\tilde{\Delta}_{\mathcal{B}}^{+}(y)$; in that case $(\mathcal{A}, \mathcal{B})$ satisfies the L-condition if and only if

$$
\operatorname{det} \tilde{\Delta}_{\mathcal{B}}^{+}(y) \neq 0 \quad \text { for all } y \in \partial \Omega .
$$

We now specialize the results of [Ro, $\S 6$ and $\S 7]$ to elliptic systems in the plane. Denote by

$$
\mathrm{BE}^{\ell, \mu}
$$

the set of elliptic boundary problems $(\mathcal{A}, \mathcal{B})$ fulfilling the L-condition such that $\mathcal{B}$ has the same order in all rows, $m_{k}=\mu(k=1, \ldots, r)$. As before, if $\left(\mathcal{A}, \mathcal{B}_{i}\right) \in \mathrm{BE}^{\ell, \mu}$, $i=1,2$, are two boundary value problems with the same elliptic operator $\mathcal{A}$ such that $\Delta_{\mathcal{B}_{1}}^{+}=\Delta_{\mathcal{B}_{2}}^{+}$, we write

$$
\left(\mathcal{A}, \mathcal{B}_{1}\right) \approx\left(\mathcal{A}, \mathcal{B}_{2}\right)
$$

Theorem 1.2. Let $\mathcal{A}_{t}, 0 \leq t \leq 1$, be a homotopy of elliptic operators in Ell ${ }^{\ell}$. If $\mathcal{B}$ is a (differential) boundary operator satisfying the L-condition relative to $\mathcal{A}=\mathcal{A}_{0}$ such that the principal part of $\mathcal{B}$ is homogeneous of degree $\ell-1$ (in all rows), then there exists a homotopy of boundary value problems $\left(\mathcal{A}_{t}, \mathcal{B}_{t}\right) \in \mathrm{BE}^{\ell, \ell-1}, 0 \leq t \leq 1$, such that $\left(\mathcal{A}, \mathcal{B}_{0}\right) \approx(\mathcal{A}, \mathcal{B})$.

Proof. This is the same proof as in [Ro, Theorem 7.3], except that it is necessary to verify that the boundary operators can be chosen to be differential operators. By the remark after [Ro, Theorem 7.3], there exist (unique) $r \times p$ matrix functions $B_{j}(t, \cdot)$ on $T^{*}(\partial \Omega) \backslash 0$ such that

$$
\sum_{j=0}^{\ell-1} B_{j}(t, \cdot) X_{ \pm}(t, \cdot) T_{ \pm}^{j}(t, \cdot)=\Delta_{\mathcal{B}}^{ \pm}
$$

Since the DN numbers of $\mathcal{B}$ are $m_{1}=\cdots=m_{r}=\ell-1$ and $t_{1}=\cdots=t_{p}=0$, we have $B_{j}\left(t, y, c \xi^{\prime}\right)=c^{\ell-1-j} B_{j}\left(t, y, \xi^{\prime}\right), j=0, \ldots, \ell-1$, for all $c$ (positive and negative). We let

$$
\mathcal{B}_{t}(y, \partial)=\sum_{j=0}^{\ell-1} B_{j}(t, y, \tau(y))(\partial / \partial \tau)^{\ell-1-j}(\partial / \partial n)^{j}
$$


which is a differential operator. Since $\Delta_{\mathcal{B}_{t}}^{ \pm}=\Delta_{\mathcal{B}}^{ \pm}$then $\left(\mathcal{A}_{t}, \mathcal{B}_{t}\right)$ satisfies the Lcondition, so we have constructed a homotopy $\left(\mathcal{A}_{t}, \mathcal{B}_{t}\right) \in \mathrm{BE}^{\ell, \ell-1}$. Also, by construction, the principal parts of $\mathcal{B}_{0}$ and $\mathcal{B}$ are the same (see [Ro, Prop. 5.2]), thus $\left(\mathcal{A}, \mathcal{B}_{0}\right) \approx(\mathcal{A}, \mathcal{B})$.

Remark 1.3. If the operators $\mathcal{A}_{t}$ and $\mathcal{B}$ have real matrix coefficients then we can find $\mathcal{B}_{t}$ also with real matrix coefficients such that $\left(\mathcal{A}_{t}, \mathcal{B}_{t}\right)$ satisfies the L-condition for all $t \in I$. Indeed,

$$
\left(X_{-}(t, \cdot), T_{-}(t, \cdot), Y_{-}(t, \cdot)\right)=\left(\overline{X_{+}(t, \cdot)}, \overline{T_{+}(t, \cdot)}, \overline{Y_{+}(t, \cdot)}\right),
$$

is a $\gamma^{-}$-spectral triple of $L_{t, .}(\lambda)$ and the solution of (8) (which does not depend on the choice of $\left.\left(X_{ \pm}, T_{ \pm}\right)\right)$can be written in the form

$$
B_{j}(t, \cdot)=2 \operatorname{Re}\left\{\Delta_{\mathcal{B}}^{+} \cdot \sum_{k=0}^{\ell-j-1} T_{+}^{k}(t, \cdot) Y_{+}(t, \cdot) A_{j+k+1}(t, \cdot)\right\},
$$

which is a real matrix function.

The same remark applies to the following theorem.

Theorem 1.4. Let $(\mathcal{A}, \mathcal{B}) \in \mathrm{BE}^{\ell, \mu}$ with $\mu \geq \ell$. Then

$$
(\mathcal{A}, \mathcal{B}) \approx\left(\mathcal{A},(\partial / \partial \tau)^{\mu-\ell+1} \mathcal{R}\right)
$$

for some (differential) boundary operator $\mathcal{R}$ such that $(\mathcal{A}, \mathcal{R}) \in \mathrm{BE}^{\ell, \ell-1}$. In fact, since $\mathcal{B}$ is a differential operator there exists $\mathcal{R}$ with $\tilde{\Delta}_{\mathcal{R}}^{ \pm}=\tilde{\Delta}_{\mathcal{B}}^{ \pm}$.

Proof. This is just a restatement of [Ro, Theorem 6.3] for systems in the plane. There exist unique $r \times p$ matrix functions $\tilde{R}_{j}$ on $\partial \Omega$ such that

$$
\sum_{j=0}^{\ell-1} R_{j}(y) X_{ \pm} T_{ \pm}^{j}=\sum_{j=0}^{\mu} B_{j}(y) X_{ \pm} T_{ \pm}^{j}, \quad y \in \partial \Omega
$$

that is,

$$
\left[\begin{array}{lll}
\tilde{R}_{0} & \ldots & \tilde{R}_{\ell-1}
\end{array}\right]=\tilde{\Delta}_{\mathcal{B}}^{+} \cdot\left[\begin{array}{lll}
\tilde{Y}_{+} & \ldots & \tilde{T}_{+}^{\ell-1} \tilde{Y}_{+}
\end{array}\right] \tilde{\mathcal{Z}}+\tilde{\Delta}_{\mathcal{B}}^{-} \cdot\left[\begin{array}{lll}
\tilde{Y}_{-} & \ldots & \tilde{T}_{-}^{\ell-1} \tilde{Y}_{-}
\end{array}\right] \tilde{\mathcal{Z}}
$$

It follows that the differential operator

$$
\mathcal{R}:=\sum_{j=0}^{\ell-1} \tilde{R}_{j}(y)(\partial / \partial \tau)^{\ell-1-j}(\partial / \partial n)^{j}
$$

satisfies (10), that is,

$$
\tilde{\Delta}_{\mathcal{R}}^{ \pm}=\tilde{\Delta}_{\mathcal{B}}^{ \pm} .
$$

Since $\mathcal{R}$ is a differential operator and $\pi \mathcal{R}$ is homogeneous of degree $\ell-1$ we have $(\mathcal{A}, \mathcal{R}) \in \mathrm{BE}^{\ell, \ell-1}$. The relation (9) follows since $\tilde{\Delta}^{ \pm}=\tilde{\Delta}_{\mathcal{R}}^{ \pm}=\tilde{\Delta}_{\mathcal{B}}^{ \pm}$.

Remark. Equation $(9)$ implies $(\mathcal{A}, \mathcal{B})$ is homotopic to $\left(\mathcal{A},(\partial / \partial \tau)^{\mu-\ell+1} \mathcal{R}\right)$ in $\mathrm{BE}^{\ell, \ell-1}$ (for instance, consider a linear homotopy). 


\section{The TOPOLOGICAL INDEX}

From now on we always assume that the region $\Omega$ is bounded and simply connected. In this section we shall define the topological index of an elliptic boundary problem (see Definition 2.5) but first we need to make a special choice of $\gamma^{+}$spectral pair for the matrix polynomial $\tilde{L}_{y}(\lambda)$ of (1-2). Let $\mathcal{A} \in \mathrm{Ell}^{\ell}$ be an elliptic operator with principal part $\pi \mathcal{A}(x, \xi)=\sum_{|\alpha|=\ell} A_{\alpha}(x) \xi^{\alpha}$, and consider the matrix polynomial

$$
L_{x}(\lambda)=\pi \mathcal{A}(x,(1, \lambda))=\sum_{j=0}^{\ell} A_{j}(x) \lambda^{j},
$$

which has coefficients $A_{j}(x)=A_{\ell-j, j}(x)$ defined for all $x \in \bar{\Omega}$ (not just the boundary). Due to ellipticity, $\operatorname{det} L_{x}(\lambda) \neq 0$ for real $\lambda$ and the leading coefficient, $A_{\ell}(x)=\pi \mathcal{A}(x,(0,1))$, is invertible. The solution space of $L_{x}\left(\frac{1}{i} \frac{d}{d t}\right) u=0$ is a direct sum $\mathfrak{M}_{L_{x}}^{+} \oplus \mathfrak{M}_{L_{x}}^{-}$, corresponding to the eigenvalues of $L_{x}(\lambda)$ with $\operatorname{Im} \lambda>0$ and $\operatorname{Im} \lambda<0$, and we let $\mathfrak{M}^{ \pm}$denote the vector bundle over $\bar{\Omega}$ with fibers $\mathfrak{M}_{L_{x}}^{ \pm}$.

Proposition 2.1. Let $\Omega$ be a bounded and simply connected region with $C^{\infty}$ boundary. Then the vector bundles $\mathfrak{M}^{+}$and $\mathfrak{M}^{-}$are trivial. Hence there exist admissible pairs $\left(X_{ \pm}, T_{ \pm}\right)$over $\bar{\Omega}$ such that $\left(X_{ \pm}(x), T_{ \pm}(x)\right)$ is a $\gamma^{ \pm}$-spectral pair of $L_{x}(\lambda)$ consisting of matrix functions with entries in $C^{\infty}(\bar{\Omega})$.

Proof. The coefficients of $\mathcal{A}$ can be extended smoothly to a neighbourhood $\Omega^{\prime} \supset \bar{\Omega}$; we may assume that $\Omega^{\prime}$ is also a simply connected region. Also, by continuity, we may assume that $\operatorname{det} \pi \mathcal{A}(x, \xi) \neq 0$ for all $x \in \Omega^{\prime}, \xi \neq 0$. Thus we obtain a vector bundle $\mathfrak{M}^{ \pm}$over $\Omega^{\prime}$ which is trivial since $\Omega^{\prime}$ is contractible. (In fact, by the Riemann mapping theorem $\Omega^{\prime}$ is conformally equivalent to the open unit disc, see $[\mathrm{Ru}$, p. 283].) Hence its restriction to $\bar{\Omega}$ is also trivial. The last statement in the proposition follows as in [Ro, Theorem 4.4].

Note that $L_{x}(\lambda)$ is defined in terms of the basis $(1,0),(0,1)$ for $\mathbb{R}^{2}$ whereas the matrix polynomial $\tilde{L}_{y}(\lambda)$ of (1-2) is defined in terms of the basis $\tau(y), n(y)$. Writing $\tau(y)=\left(\tau_{1}(y), \tau_{2}(y)\right)$ and $n(y)=\left(-\tau_{2}(y), \tau_{1}(y)\right)$ we have

$$
\begin{aligned}
L_{y}(\lambda) & =\pi \mathcal{A}(y, \tau(y)+\lambda n(y)) \\
& =\sum_{j=0}^{\ell} A_{j}(y)\left(\tau_{1}(y)-\lambda \tau_{2}(y)\right)^{\ell-j}\left(\tau_{2}(y)+\lambda \tau_{1}(y)\right)^{j},
\end{aligned}
$$

where $\varphi^{-1}(\lambda)=\left(\tau_{2}+\lambda \tau_{1}\right) /\left(\tau_{1}-\lambda \tau_{2}\right)$. Let $\left(X_{ \pm}(y), T_{ \pm}(y)\right)$ be a $\gamma^{ \pm}$-spectral pair of $L_{y}(\lambda)$. Since $\varphi$ maps the upper half-plane to itself, then one can show that

$$
\begin{aligned}
\tilde{X}_{ \pm}(y) & =X_{ \pm}(y), \\
\tilde{T}_{ \pm}(y) & =\left(\tau_{1}(y) T_{ \pm}(y)-\tau_{2}(y) I\right)\left(\tau_{2}(y) T_{ \pm}(y)+\tau_{1}(y) I\right)^{-1}
\end{aligned}
$$

is a $\gamma^{ \pm}$-spectral pair of $\tilde{L}_{y}(\lambda)$ (see [WRL, $\left.\S 2.4\right]$ ).

In the next proposition we assume that $(\mathcal{A}, \mathcal{B}) \in \mathrm{BE}^{\ell, \mu}$, that is, the principal part of the boundary operator $\mathcal{B}$ is homogeneous of degree $\mu$ in each row, $\pi \mathcal{B}(y, \xi)=$ $\sum_{|\alpha|=\mu} B_{\alpha}(y) \xi^{\alpha}$. Analogous to the considerations for $L_{x}(\lambda)$, there is the following 
$r \times p$ matrix polynomial

$$
B_{y}(\lambda)=\pi \mathcal{B}(y,(1, \lambda))=\sum_{j=0}^{\mu} B_{j}(y) \lambda^{j}, \quad y \in \partial \Omega,
$$

where $B_{j}(y)=B_{\mu-j, j}(y)$. We let

$$
\Delta_{\mathcal{B}}^{ \pm}(y)=\sum_{j=0}^{\mu} B_{j}(y) X_{ \pm}(y) T_{ \pm}^{j}(y)
$$

where $\left(X_{ \pm}(y), T_{ \pm}(y)\right)$ is a $\gamma^{ \pm}$-spectral pair of $L_{y}(\lambda), y \in \partial \Omega$. Note: It is important that the reader distinguish the matrix functions $\Delta_{\mathcal{B}}^{ \pm}(y)$ from $\Delta_{\mathcal{B}}^{+}\left(y, \xi^{\prime}\right)$ which was defined in (1-5).

Proposition 2.2. Let $\left(X_{ \pm}(y), T_{ \pm}(y)\right)$ be $\gamma^{ \pm}$-spectral pairs of $L_{y}(\lambda)$, and $\left(\tilde{X}_{ \pm}(y)\right.$, $\left.\tilde{T}_{ \pm}(y)\right)$ be the corresponding $\gamma^{ \pm}$-spectral pairs of $\tilde{L}_{y}(\lambda)$ as defined by the equations (2). Let $\mathcal{B}$ be a boundary operator whose principal part is homogeneous of degree $\mu$. Then

$$
\tilde{\Delta}_{\mathcal{B}}^{ \pm}(y)=\Delta_{\mathcal{B}}^{ \pm}(y) \cdot\left(\tau_{1}(y) I+\tau_{2}(y) T_{ \pm}(y)\right)^{-\mu}, \quad y \in \partial \Omega .
$$

Hence $\operatorname{det} \Delta_{\mathcal{B}}^{ \pm}(y) \neq 0$ if and only if $\operatorname{det} \tilde{\Delta}_{\mathcal{B}}^{ \pm}(y) \neq 0$.

The proof is left to the reader.

Corollary 2.3. Let the hypotheses be as in Proposition 2.2. Then the following are equivalent for all $y \in \partial \Omega$ :

(i) $u \mapsto \tilde{B}_{y}\left(\frac{1}{i} \frac{d}{d t}\right) u_{\mid t=0}$ from $\mathfrak{M}_{\tilde{L}_{y}}^{ \pm}$to $\mathbb{C}^{r}$ is invertible;

(ii) $u \mapsto B_{y}\left(\frac{1}{i} \frac{d}{d t}\right) u_{\mid t=0}$ from $\mathfrak{M}_{L_{y}}^{ \pm}$to $\mathbb{C}^{r}$ is invertible.

Proof. In view of [Ro, Theorem 3.1], (i) and (ii) are equivalent to:

$$
\left(\mathrm{i}^{\prime}\right) \quad \operatorname{det} \tilde{\Delta}_{\mathcal{B}}^{ \pm}(y) \neq 0, \quad\left(\mathrm{ii}^{\prime}\right) \quad \operatorname{det} \Delta_{\mathcal{B}}^{ \pm}(y) \neq 0,
$$

and $\left(\mathrm{i}^{\prime}\right)$ and $\left(\mathrm{ii}^{\prime}\right)$ are equivalent by Proposition 2.2.

Proposition 2.1 has the following important consequence.

Theorem 2.4. Let $\Omega \subset \mathbb{R}^{2}$ be a bounded and simply connected region with $C^{\infty}$ boundary. A properly elliptic operator $\mathcal{A}$ in $\bar{\Omega}$ always has a differential boundary operator satisfying the L-condition.

Proof. Let $\mathcal{A} \in \mathrm{Ell}^{\ell}$, choose $\left(X_{ \pm}(x), T_{ \pm}(x)\right)$ as in Proposition 2.1, and then define the spectral pair (2). There is a natural differential boundary operator associated with the spectral pair $\left(\tilde{X}_{ \pm}(y), \tilde{T}_{ \pm}(y)\right)$ since the system

$$
\sum_{j=0}^{\ell-1} \tilde{B}_{j}(y) \tilde{X}_{ \pm} \tilde{T}_{ \pm}^{j}=I_{r}
$$

where $I_{r}$ denotes the $r \times r$ identity matrix, has the unique solution

$$
\left[\begin{array}{lll}
\tilde{B}_{0} & \ldots & \tilde{B}_{\ell-1}
\end{array}\right]=\left[\begin{array}{lll}
\tilde{Y}_{+} & \ldots & \tilde{T}_{+}^{\ell-1} \tilde{Y}_{+}
\end{array}\right] \tilde{\mathcal{Z}}+\left[\begin{array}{lll}
\tilde{Y}_{-} & \ldots & \tilde{T}_{-}^{\ell-1} \tilde{Y}_{-}
\end{array}\right] \tilde{\mathcal{Z}} .
$$

The boundary operator

$$
\mathcal{B}^{o}=\sum_{j=0}^{\ell-1} \tilde{B}_{j}(y)(\partial / \partial \tau)^{\ell-1-j}(\partial / \partial n)^{j}
$$


therefore satisfies $\tilde{\Delta}_{\mathcal{B}^{o}}^{ \pm} \equiv I_{r}$ and we have $\left(\mathcal{A}, \mathcal{B}^{o}\right) \in \mathrm{BE}^{\ell, \ell-1}$.

Let $S^{1}$ denote the unit circle, and let $f$ be a continuous nonvanishing complexvalued function on $S^{1}$, i.e. $f \in C\left(S^{1}, \mathbb{C}^{*}\right)$ where $\mathbb{C}^{*}=\mathbb{C} \backslash 0$. Then

$$
\frac{f\left(e^{i t}\right)}{\left|f\left(e^{i t}\right)\right|}=e^{i \theta(t)}
$$

for some real-valued $\theta \in C([0,2 \pi])$, and the degree (or winding number) of $f$ is defined to be

$$
\operatorname{deg} f=[\arg f(z)]_{S^{1}}=(\theta(2 \pi)-\theta(0)) / 2 \pi .
$$

Note that $\theta$ defines a continuous function on $S^{1}$ only when $\operatorname{deg} f=0$, i.e. $\theta(2 \pi)=$ $\theta(0)$. The degree has the following multiplicative property

$$
\operatorname{deg}(f \cdot g)=\operatorname{deg} f+\operatorname{deg} g .
$$

In particular, $\operatorname{deg}(1 / f)=-\operatorname{deg} f$. The degree of a function is locally constant; in fact if

$$
\max _{z \in S^{1}}|g(z)-f(z)|<\max _{z \in S^{1}}|f(z)|
$$

then $\operatorname{deg} g=\operatorname{deg} f$. Let $f, g \in C\left(S^{1}, \mathbb{C}^{*}\right)$. Then $f$ and $g$ are said to be homotopic if there exists a continuous function $F: S^{1} \times I \rightarrow \mathbb{C}^{*}$ such that $F(\cdot, 0)=f$ and $F(\cdot, 1)=g$, where $I$ denotes the unit interval $[0,1]$. If $f$ and $g$ are homotopic we write $f \sim g$. It is evident that if $f_{1} \sim f_{2}$ and $g_{1} \sim g_{2}$ then $f_{1} \cdot g_{1} \sim f_{2} \cdot g_{2}$. A function $f \in C\left(S^{1}, \mathbb{C}^{*}\right)$ is said to be null-homotopic if $f \sim 1$, i.e. if it is homotopic to the constant function 1 . A function $f \in C\left(S^{1}, \mathbb{C}^{*}\right)$ is null-homotopic if and only if it has degree 0 . Any nonzero constant function is obviously null-homotopic. Also, any real-valued function $f \in C\left(S^{1}, \mathbb{R}^{*}\right)$ is null-homotopic.

As in the proof of Proposition 2.1, there exists an orientation-preserving diffeomorphism $\varphi$ from the closed unit disc to $\bar{\Omega}$. Then the degree of a nonvanishing continuous function $f \in C(\partial \Omega)$ is defined to be $\operatorname{deg}(f \circ \varphi)$, where the degree on $S^{1}$ is defined as above. Note that the degree is independent of the choice of $\varphi$; in the sequel we also use the notation

$$
\operatorname{deg}(f \circ \varphi)=:[\arg f(y)]_{\partial \Omega} .
$$

Now we consider matrix functions. Let $M: S^{1} \rightarrow \mathrm{GL}_{r}(\mathbb{C})$ be a continuous matrix function, where $\mathrm{GL}_{r}(\mathbb{C})$ denotes the group of invertible $r \times r$ matrices with complex entries. The degree of $M$ is defined to be the degree of the complex-valued function $\operatorname{det} M$,

$$
\operatorname{deg} M=[\arg \operatorname{det} M(z)]_{S^{1}} .
$$

As with scalar functions, two continuous $r \times r$ matrix functions on $S^{1}$ with invertible values are homotopic if and only if their degrees coincide.

Finally, if $M$ is a continuous $r \times r$ matrix function on $\partial \Omega$ with nonvanishing determinant then the degree of $M$ is defined to be the degree of the complex-valued function $\operatorname{det} M$, i.e. $[\arg \operatorname{det} M(y)]_{\partial \Omega}$.

Now we write down the definition of the topological index of an elliptic boundary problem. This requires us to choose a $C^{\infty}$ matrix $\gamma^{ \pm}$-spectral pair $\left(X_{ \pm}(x), T_{ \pm}(x)\right)$ of $L_{x}(\lambda), x \in \bar{\Omega}$, as in Proposition 2.1. The definition, however, does not actually depend on the choice of such a pair (see Lemma 2.7). 
Definition 2.5. The topological index of a boundary value problem $(\mathcal{A}, \mathcal{B})$ is defined as follows:

$$
\operatorname{ind}_{s}(\mathcal{A}, \mathcal{B})=r(2-\ell)-\frac{1}{2 \pi}\left[\arg \operatorname{det} \tilde{\Delta}_{\mathcal{B}}^{+}(y)\right]_{\partial \Omega}+\frac{1}{2 \pi}\left[\arg \operatorname{det} \tilde{\Delta}_{\mathcal{B}}^{-}(y)\right]_{\partial \Omega}
$$

where $\left(X_{ \pm}(x), T_{ \pm}(x)\right), x \in \bar{\Omega}$, is chosen as in Proposition 2.1, and then $\left(\tilde{X}_{ \pm}(y), \tilde{T}_{ \pm}(y)\right)$, $y \in \partial \Omega$, is defined by the transformation $(2)$ and $\tilde{\Delta}_{\mathcal{B}}^{ \pm}(y)$ is defined by $(1-4)$.

At first sight it might appear that the definition of $\operatorname{ind}_{s}(\mathcal{A}, \mathcal{B})$ depends only on the value of the coefficients of $\mathcal{A}$ on the boundary $\partial \Omega$, but in fact the choice of $\left(X_{+}, T_{+}\right)$and hence of $\left(\tilde{X}_{+}, \tilde{T}_{+}\right)$depends on the coefficients throughout the region $\bar{\Omega}$. If the operators $\mathcal{A}$ and $\mathcal{B}$ have real coefficients, we may assume $\tilde{\Delta}_{-}(y)={\overline{\Delta_{+}}}(y)$ (see Remark 1.1) and then

$$
\operatorname{ind}_{s}(\mathcal{A}, \mathcal{B})=r(2-\ell)-\frac{1}{\pi}\left[\arg \operatorname{det} \tilde{\Delta}_{\mathcal{B}}^{+}(y)\right]_{\partial \Omega}
$$

The next two lemmas show that $\operatorname{ind}_{s}(\mathcal{A}, \mathcal{B})$ is well-defined, independent of the choice of $\gamma^{ \pm}$-spectral pairs.

Lemma 2.6. If $(\mathcal{A}, \mathcal{B}) \in \mathrm{BE}^{\ell, \mu}$, i.e. the principal part of the boundary operator $\mathcal{B}$ is homogeneous of degree $\mu$ in all rows, then the quantity

$$
\left[\arg \operatorname{det} \Delta_{\mathcal{B}}^{ \pm}(y)\right]_{\partial \Omega}
$$

is independent of the choice of a $C^{\infty}$ matrix $\gamma^{ \pm}$-spectral pair $\left(X_{ \pm}(x), T_{ \pm}(x)\right)$ of $L_{x}(\lambda), x \in \bar{\Omega}$, where $\Delta_{\mathcal{B}}^{ \pm}(y)$ is defined by $(3)$.

Proof. Let $\left(X_{ \pm}^{o}(x), T_{ \pm}^{o}(x)\right)$ be another $\gamma^{ \pm}$-spectral pair of $L_{x}(\lambda), x \in \bar{\Omega}$, consisting of matrix functions with entries in $C^{\infty}(\bar{\Omega})$. It can be shown that $\gamma^{ \pm}$-spectral pairs are unique up to similarity; in fact it follows from [WRL, Proposition 2.12] that

$$
X_{ \pm}^{o}(x)=X_{ \pm}(x) M(x), \quad T_{ \pm}^{o}(x)=M^{-1}(x) T_{ \pm}(x) M(x)
$$

for some matrix function $M$ with entries in $C^{\infty}(\bar{\Omega})$ such that $\operatorname{det} M(x) \neq 0$ for all $x \in \bar{\Omega}$. For the sake of brevity we write $\Delta_{ \pm}=\Delta_{\mathcal{B}}^{ \pm}$. Then

$$
\Delta_{ \pm}^{o}(y)=\Delta_{ \pm}(y) M(y) \quad \text { for all } y \in \partial \Omega
$$

and it follows that

$$
\left[\arg \operatorname{det} \Delta_{ \pm}^{o}(y)\right]_{\partial \Omega}=\left[\arg \operatorname{det} \Delta_{ \pm}(y)\right]_{\partial \Omega}+[\arg \operatorname{det} M(y)]_{\partial \Omega} .
$$

As in the proof of Proposition 2.1, $\bar{\Omega}$ is contractible to a point $x_{0} \in \bar{\Omega}$, whence the function $\operatorname{det} M(x), x \in \bar{\Omega}$, is homotopic to a constant. Its restriction to $\partial \Omega$ is therefore homotopic to a constant function, thus

$$
[\arg \operatorname{det} M(y)]_{\partial \Omega}=0
$$

as was to be shown.

The following lemma is another version of Lemma 2.6 for which there is no special assumption on the orders of the rows of the boundary operator.

Lemma 2.7. Let the $\gamma^{ \pm}$-spectral pair $\left(\tilde{X}_{ \pm}(y), \tilde{T}_{ \pm}(y)\right)$ of $\tilde{L}_{y}(\lambda)$ be defined by the transformation (2), and then define $\tilde{\Delta}_{\mathcal{B}}^{ \pm}(y)$ by (1-4). Then the quantity

$$
\left[\arg \operatorname{det} \tilde{\Delta}_{\mathcal{B}}^{ \pm}(y)\right]_{\partial \Omega}
$$

is independent of the choice of the $C^{\infty}$ matrix $\gamma^{ \pm}$-spectral pair $\left(X_{ \pm}(x), T_{ \pm}(x)\right)$ of $L_{x}(\lambda), x \in \bar{\Omega}$. 
Proof. The boundary operator has the form (1-1b). Now we operate on $\mathcal{B}$ with tangential derivatives to make the order the same in each row:

$$
\mathcal{B}^{\prime}=\left[(\partial / \partial \tau)^{\mu-m_{k}} \sum_{|\alpha| \leq m_{k}} b_{k}(y) \partial^{\alpha}\right]_{k=1}^{r},
$$

where $\mu=\max _{k} m_{k}$ and $\partial / \partial \tau=\tau_{1}(y) \cdot \partial / \partial x_{1}+\tau_{2}(y) \cdot \partial / \partial x_{2}$. Let us write $\tilde{\Delta}_{ \pm}=\tilde{\Delta}_{\mathcal{B}}^{ \pm}$ and $\tilde{\Delta}_{ \pm}^{\prime}=\tilde{\Delta}_{\mathcal{B}^{\prime}}^{ \pm}$. For the matrix polynomials associated with the boundary operators $\mathcal{B}^{\prime}$ and $\mathcal{B}$, we clearly have $\tilde{B}_{y}^{\prime}(\lambda)=\tilde{B}_{y}(\lambda)$. Thus $\tilde{\Delta}_{ \pm}^{\prime}(y)=\tilde{\Delta}_{ \pm}(y)$, which has nonzero determinant for all $y \in \partial \Omega$, so $\mathcal{B}^{\prime}$ also satisfies the L-condition. Due to Proposition 2.2 we have

$$
\left[\arg \operatorname{det} \tilde{\Delta}_{ \pm}(y)\right]_{\partial \Omega}=\left[\arg \operatorname{det} \Delta_{ \pm}^{\prime}(y)\right]_{\partial \Omega}-\mu\left[\arg \operatorname{det}\left(\tau_{1}(y) I+\tau_{2}(y) T_{ \pm}(y)\right)\right]_{\partial \Omega}
$$

Since the spectrum of $T_{+}$lies in the upper half-plane, the same is true of $t i I+(1-t) T_{+}$for $0 \leq t \leq 1$, and therefore

$$
H(y, t)=\tau_{1}(y) I+\tau_{2}(y)\left\{t i I+(1-t) T_{+}(y)\right\}, \quad y \in \partial \Omega, \quad t \in I,
$$

defines a homotopy $\partial \Omega \times I \rightarrow \mathrm{GL}_{r}(\mathbb{C})$. The winding number of a continuous function is locally constant, whence

$$
\frac{1}{2 \pi}\left[\arg \operatorname{det}\left(\tau_{1}(y) I+\tau_{2}(y) T_{+}(y)\right)\right]_{\partial \Omega}=\frac{1}{2 \pi}\left[\arg \operatorname{det}\left(\tau_{1}(y) I+\tau_{2}(y) i I\right)\right]_{\partial \Omega}=r .
$$

Similarly, since the spectrum of $T_{-}$lies in the lower half-plane, we find that

$$
\frac{1}{2 \pi}\left[\arg \operatorname{det}\left(\tau_{1}(y) I+\tau_{2}(y) T_{-}(y)\right)\right]_{\partial \Omega}=-r .
$$

Thus

$$
\frac{1}{2 \pi}\left[\arg \operatorname{det} \tilde{\Delta}_{+}(y)\right]_{\partial \Omega}=\frac{1}{2 \pi}\left[\arg \operatorname{det} \Delta_{+}^{\prime}(y)\right]_{\partial \Omega}-r \mu
$$

and

$$
\frac{1}{2 \pi}\left[\arg \operatorname{det} \tilde{\Delta}_{-}(y)\right]_{\partial \Omega}=\frac{1}{2 \pi}\left[\arg \operatorname{det} \Delta_{-}^{\prime}(y)\right]_{\partial \Omega}+r \mu .
$$

According to Lemma 2.6, the quantity $\left[\arg \operatorname{det} \Delta_{ \pm}^{\prime}(y)\right]_{\partial \Omega}$ is independent of the choice of $\left(X_{ \pm}, T_{ \pm}\right)$; hence the same is true of $\left[\arg \operatorname{det} \tilde{\Delta}_{ \pm}(y)\right]_{\partial \Omega}$.

\section{A SIMPLE FORM FOR FIRST-ORDER ELLIPTIC SYSTEMS WITH REAL COEFFICIENTS}

Consider a first-order elliptic boundary value problem (BVP) in the plane

$$
\begin{gathered}
\frac{\partial u}{\partial x_{2}}-A(x) \frac{\partial u}{\partial x_{1}}+A_{0}(x) u=f(x), \quad x=\left(x_{1}, x_{2}\right) \in \Omega, \\
B(y) u=g(y), \quad y \in \partial \Omega
\end{gathered}
$$

where $A(x)$ and $A_{0}(x)$ are real $2 r \times 2 r$ matrix functions with entries in $C^{\infty}(\bar{\Omega})$, and $B(y)$ is a real $r \times 2 r$ matrix function with entries in $C^{\infty}(\partial \Omega)$. The region $\Omega \subset \mathbb{R}^{2}$ is bounded and simply connected, with smooth boundary.

Any elliptic operator $\mathcal{A}$ where the principal part is homogeneous of order 1 can be written in the form (1a). Indeed, the principal part $\pi \mathcal{A}(x, \xi)$ is invertible for all $x \in \bar{\Omega}, 0 \neq \xi \in \mathbb{R}^{2}$, and letting $\xi=(0,1)$ we see that the coefficient of $\partial / \partial x_{2}$ is invertible for all $x \in \bar{\Omega}$. 
The BVP (1a), (1b) is elliptic if $\operatorname{det}(I \lambda-A(x)) \neq 0$ for all $x \in \bar{\Omega}, \lambda \in \mathbb{R}$, and satisfies the Lopatinskii condition if the $r \times 2 r$ Lopatinskii matrix

$$
B(y) \cdot \int_{\gamma^{+}}(I \lambda-A(y))^{-1} d \lambda
$$

has rank $r$ for all $y \in \partial \Omega$, where the integral is taken along a simple, closed contour $\gamma^{+}$in the half-plane $\operatorname{Im} \lambda>0$ containing the zeros of $\operatorname{det}(I \lambda-A(y))$ there.

In that case, the linear operator

$$
(\mathcal{A}, \mathcal{B}): W_{2}^{1}\left(\Omega, \mathbb{R}^{2 r}\right) \longrightarrow L_{2}\left(\Omega, \mathbb{R}^{2 r}\right) \times W_{2}^{1 / 2}\left(\partial \Omega, \mathbb{R}^{r}\right),
$$

defined by $u \mapsto\left(\partial u / \partial x_{2}-A \partial u / \partial x_{1}+A_{0} u, B u\right)$, is Fredholm. Since the index of Fredholm operators in Banach spaces is locally constant, then ind $(\mathcal{A}, \mathcal{B})$ depends only on the principal part of $\mathcal{A}$, that is,

$$
\operatorname{ind}(\mathcal{A}, \mathcal{B})=\operatorname{ind}(\pi \mathcal{A}, \mathcal{B})
$$

because one can replace the coefficient $A_{0}(x)$ by $t A_{0}(x), 0 \leq t \leq 1$, giving a homotopy between $(\mathcal{A}, \mathcal{B})$ and $(\pi \mathcal{A}, \mathcal{B})$. Thus, for the purpose of determining the index we may assume that $A_{0}=0$.

Remark. Since $\mathcal{A}$ and $\mathcal{B}$ have real matrix coefficients, there is no loss of generality in considering solutions $u$ which are real vector functions rather than complex (just take real and imaginary parts of $u$ ). This does not affect the index.

The following lemma is used in $\S 4$ to transform the $2 r \times 2 r$ real system (1a), (1b) into a generalized Riemann-Hilbert problem (an $r \times r$ complex system). The index for this new problem is easy to determine since it is homotopic to a diagonal system of $r$ (scalar) Riemann-Hilbert problems. Then we derive a formula for the index of $(\mathcal{A}, \mathcal{B})$.

Lemma 3.1. Let $A$ be a $2 r \times 2 r$ real matrix such that $\operatorname{sp}(A) \cap \mathbb{R}=\emptyset$. Then there exists a real invertible $2 r \times 2 r$ matrix $Q$ such that

$$
Q^{-1} A Q=\left(\begin{array}{cc}
A_{1} & -A_{2} \\
A_{2} & A_{1}
\end{array}\right)
$$

where $A_{1}, A_{2}$ are real $r \times r$ matrices such that $\operatorname{sp}\left(A_{1}+i A_{2}\right)$ lies in the upper halfplane.

Proof. Write $\mathbb{C}^{2 r}=\mathfrak{M}_{+} \oplus \mathfrak{M}_{-}$, where $\mathfrak{M}_{ \pm}$are the invariant subspaces of $A$ corresponding to the eigenvalues in the upper and lower half-planes, respectively. Since $A$ has real coefficients, there is a one-to-one correspondence between $\mathfrak{M}_{+}$and $\mathfrak{M}_{-}$ given by conjugation.

Let $X_{+}$be a $2 r \times r$ matrix whose columns form a basis of $\mathfrak{M}_{+}$; then the columns of $\bar{X}_{+}$form a basis of $\mathfrak{M}_{-}=\overline{\mathfrak{M}}_{+}$. Let $T_{+}$be the unique $r \times r$ matrix such that $A X_{+}=X_{+} T_{+}$. Then,

$$
\begin{aligned}
A\left[\begin{array}{ll}
X_{+} & \bar{X}_{+}
\end{array}\right] & =\left[\begin{array}{ll}
A X_{+} & A \bar{X}_{+}
\end{array}\right] \\
& =\left[\begin{array}{ll}
X_{+} T_{+} & \bar{X}_{+} \bar{T}_{+}
\end{array}\right] \\
& =\left[\begin{array}{ll}
X_{+} & \bar{X}_{+}
\end{array}\right]\left(\begin{array}{ll}
T_{+} & \\
& \bar{T}_{+}
\end{array}\right) .
\end{aligned}
$$

Thus the columns of the $2 r \times 2 r$ matrix $\left[X_{+} \bar{X}_{+}\right]$form a basis of $\mathbb{C}^{2 r}$ and relative to this basis $A$ is block diagonal. 
Now write $T_{+}=A_{1}+i A_{2}$, where $A_{1}, A_{2}$ are real $r \times r$ matrices. By Lemma 6.1 (see below), we have

$$
\left(\begin{array}{cc}
A_{1} & -A_{2} \\
A_{2} & A_{1}
\end{array}\right)=\left(\begin{array}{cc}
I & I \\
-i I & i I
\end{array}\right)\left(\begin{array}{cc}
T_{+} & \\
& \bar{T}_{+}
\end{array}\right)\left(\begin{array}{cc}
I & I \\
-i I & i I
\end{array}\right)^{-1},
$$

where $I$ is the $r \times r$ identity matrix. Let

$$
Q=\left[\begin{array}{ll}
X_{+} & \bar{X}_{+}
\end{array}\right]\left(\begin{array}{cc}
I & I \\
-i I & i I
\end{array}\right)^{-1}
$$

then (2) follows from (3) and (4). Note that the entries of $Q$ are real since

$$
Q=\left[\begin{array}{ll}
X_{+} & \bar{X}_{+}
\end{array}\right] \cdot \frac{1}{2}\left(\begin{array}{cc}
I & i I \\
I & -i I
\end{array}\right)=\left[\begin{array}{ll}
\operatorname{Re} X_{+} & -\operatorname{Im} \bar{X}_{+}
\end{array}\right]
$$

where $\operatorname{Re} X_{+}=\frac{1}{2}\left(X_{+}+\bar{X}_{+}\right)$and $\operatorname{Im} X_{+}=\frac{1}{2 i}\left(X_{+}-\bar{X}_{+}\right)$are the real and imaginary parts of $X_{+}$, respectively. Also $\operatorname{sp}\left(T_{+}\right)=\operatorname{sp}\left(A_{\mid \mathfrak{M}_{+}}\right)$which lies in the upper halfplane.

Remark. It should be noted that, as usual, in linear algebra the notation $\operatorname{sp}(A)$ in the statement of Lemma 3.1 refers to the spectrum of the matrix polynomial $L(\lambda)=I \lambda-A$. In the proof of Lemma $3.1,\left(X_{+}, T_{+}\right)$is actually a $\gamma^{+}$-spectral pair of $L(\lambda)$. Moreover, if we define

$$
X=\left[\begin{array}{ll}
X_{+} & \bar{X}_{+}
\end{array}\right], \quad T=\left(\begin{array}{cc}
T_{+} & \\
& \bar{T}_{+}
\end{array}\right), \quad Y=\left(\begin{array}{c}
Y_{+} \\
\bar{Y}_{+}
\end{array}\right)
$$

then $(X, T, Y)$ is a standard triple of $L(\lambda)$. This follows from the next lemma.

Lemma 3.2. Let $A$ be any $n \times n$ matrix. Let $X, T$ and $Y$ also be $n \times n$ matrices. Then $(X, T, Y)$ is a standard triple of $L(\lambda)=I \lambda-A$ if and only if $X$ is invertible, $A X=X T$, and $X^{-1}=Y$.

Proof. See [Ro, Definition 2.15].

Lemma 3.3. Let $A_{1}, A_{2}$ be real $r \times r$ matrices such that $\operatorname{sp}\left(A_{1}+i A_{2}\right)$ lies in the upper half-plane, and consider the $2 r \times 2 r$ matrix polynomial $L(\lambda)=I \lambda-A$, where

$$
A=\left(\begin{array}{cc}
A_{1} & -A_{2} \\
A_{2} & A_{1}
\end{array}\right) .
$$

Then $\operatorname{sp}(L) \cap \mathbb{R}=\emptyset$ and $L(\lambda)$ has the following standard triple:

$$
X=\left(\begin{array}{cc}
I & I \\
-i I & i I
\end{array}\right), \quad T=\left(\begin{array}{cc}
A_{1}+i A_{2} & \\
& A_{1}-i A_{2}
\end{array}\right), \quad Y=\frac{1}{2}\left(\begin{array}{cc}
I & i I \\
I & -i I
\end{array}\right) .
$$

Proof. Let $T_{+}=A_{1}+i A_{2}$. In view of (4) we have

$$
\operatorname{det} L(\lambda)=\left|\operatorname{det}\left(I \lambda-T_{+}\right)\right|^{2} \neq 0
$$

for real $\lambda$. By direct computation we also have $A X=X T$ and $X^{-1}=Y$, so $(X, T, Y)$ is a standard triple of $L(\lambda)$.

Corollary 3.4. Under the hypotheses of Lemma 3.3, $L(\lambda)$ has the following $\gamma^{+}$spectral triple:

$$
\left(X_{+}, T_{+}, Y_{+}\right)=\left(\left(\begin{array}{c}
I \\
-i I
\end{array}\right), A_{1}+i A_{2}, \frac{1}{2}\left[\begin{array}{ll}
I & i I
\end{array}\right]\right) .
$$


We return now to the BVP (1a), (1b). Let $L_{x}(\lambda)=I \lambda-A(x)$. By Proposition 2.1 there exists a $\gamma^{+}$-spectral pair $\left(X_{+}(x), T_{+}(x)\right)$ of $L_{x}(\lambda)$ consisting of matrices with entries that are in $C^{\infty}(\bar{\Omega})$, where the dimensions of $X_{+}$and $T_{+}$are $2 r \times r$ and $r \times r$, respectively. Then $A(x) X_{+}(x)=X_{+}(x) T_{+}(x)$ and if we let

$$
Q(x)=\left[\begin{array}{ll}
X_{+}(x) & \overline{X_{+}(x)}
\end{array}\right]\left(\begin{array}{cc}
I & I \\
-i I & i I
\end{array}\right)^{-1},
$$

the proof of Lemma 3.1 shows that

$$
Q^{-1}(x) A(x) Q(x)=\left(\begin{array}{cc}
A_{1}(x) & -A_{2}(x) \\
A_{2}(x) & A_{1}(x)
\end{array}\right), \quad x \in \bar{\Omega},
$$

where $A_{1}, A_{2}$ are $C^{\infty}$ real $r \times r$ matrix functions on $\bar{\Omega}$ and the spectrum of $T_{+}(x)=$ $A_{1}(x)+i A_{2}(x)$ lies in the upper half-plane for all $x \in \bar{\Omega}$. Let $u=Q \cdot v$, then BVP (1) takes the form

$$
\begin{gathered}
\frac{\partial v}{\partial x_{2}}-\tilde{A} \frac{\partial v}{\partial x_{1}}+\tilde{A}_{0} v=f(x), \quad x \in \Omega, \\
\tilde{B}(y) v=g(y), \quad y \in \partial \Omega,
\end{gathered}
$$

where $\tilde{A}_{0}=Q^{-1} A_{0} Q+\partial Q / \partial x_{2}-A \partial Q / \partial x_{1}, \tilde{A}=Q^{-1} A Q, \tilde{B}=B Q$ and $\tilde{f}=Q^{-1} f$. For the purpose of determining an index formula, we may of course assume that the lower order terms in $(\tilde{1})$ are 0 , that is, $\tilde{A}_{0}=0$.

Let $(\mathcal{A}, \mathcal{B})$ and $(\tilde{\mathcal{A}}, \tilde{\mathcal{B}})$ denote the boundary value problems $(1)$ and $(\tilde{1})$, respectively. Let us compare the L-condition for $(\mathcal{A}, \mathcal{B})$ with that for $(\tilde{\mathcal{A}}, \tilde{\mathcal{B}})$. First of all,

$$
\int_{\gamma^{+}} \tilde{B}(y)(I \lambda-\tilde{A}(y))^{-1} d \lambda=\int_{\gamma^{+}} B(y)(I \lambda-A(y))^{-1} d \lambda \cdot Q(y) .
$$

From this equation we see that $(\mathcal{A}, \mathcal{B})$ satisfies the L-condition if and only if $(\tilde{\mathcal{A}}, \tilde{\mathcal{B}})$ satisfies the L-condition. Further, observe that (6) may be written as

$$
\tilde{B}(y) \tilde{P}_{+}(y)=B(y) P_{+}(y) Q(y),
$$

where $\tilde{P}_{+}(y)$ and $P_{+}(y)$ are the Riesz projectors for $\tilde{A}(y)$ and $A(y)$ with respect to the eigenvalues in the upper half-plane. Then multiplying both sides of this equation on the right by $\left(\begin{array}{cc}I & I \\ -i I & i I\end{array}\right)$ and using (5) we obtain

$$
\tilde{B}(y) \tilde{P}_{+}(y) \cdot\left[\begin{array}{ll}
\tilde{X}_{+}(y) & \overline{X_{+}(y)}
\end{array}\right]=B(y) P_{+}(y) \cdot\left[\begin{array}{ll}
X_{+}(y) & \overline{X_{+}(y)}
\end{array},\right.
$$

where $\tilde{X}_{+}(y)=\left(\begin{array}{c}I \\ -i I\end{array}\right)$; see Corollary 3.4. Hence

$$
\tilde{B}(y) \cdot\left[\tilde{X}_{+}(y) \quad 0\right]=B(y) \cdot\left[X_{+}(y) \quad 0\right]
$$

that is,

$$
\tilde{B}(y) \tilde{X}_{+}(y)=B(y) X_{+}(y) .
$$

Writing $\tilde{B}(y)=\left[B_{1}(y) \quad B_{2}(y)\right]$, where $B_{1}, B_{2}$ are real $r \times r$ matrix functions, then $\tilde{B}(y) \tilde{X}_{+}(y)$ equals $B_{1}(y)-i B_{2}(y)$, and we have proved the following lemma. 
Lemma 3.5. The boundary value problem $(\mathcal{A}, \mathcal{B})$ satisfies the $L$-condition if and only if $(\tilde{\mathcal{A}}, \tilde{\mathcal{B}})$ also satisfies the L-condition. This condition can be stated in two equivalent ways:

$$
\operatorname{det} B(y) X_{+}(y) \neq 0 \quad \text { for all } y \in \partial \Omega
$$

or

$$
\operatorname{det}\left(B_{1}(y)-i B_{2}(y)\right) \neq 0 \quad \text { for all } y \in \partial \Omega,
$$

where $\tilde{B}(y)=\left[B_{1}(y) \quad B_{2}(y)\right]$.

The first condition can be written out in a more explicit manner. Let $q_{k}(y), k=$ $1, \ldots, r$, denote the columns of $X_{+}(y)$ and let $b_{j}(y), j=1, \ldots, r$, be the rows of $B(y)$. Then the BVP (1) satisfies the L-condition if and only if

$$
\operatorname{det}\left[b_{j}(y) \cdot q_{k}(y)\right]_{j, k=1}^{r} \neq 0 \quad \text { for all } y \in \partial \Omega .
$$

By taking complex conjugates, we could also write the second condition of Lemma 3.5 as

$$
\operatorname{det}\left(B_{1}(y)+i B_{2}(y)\right) \neq 0 .
$$

\section{The INDEX FORMUlA FOR FIRST-ORDER ELLIPTIC SYSTEMS WITH REAL COEFFICIENTS}

In this section we prove an index formula due to Vol'pert for first-order elliptic systems, by starting from the formula for the index of the Riemann-Hilbert problem (see the introduction).

Let $A_{i}$ and $B_{i}(i=1,2)$ be smooth $r \times r$ real matrix functions on $\bar{\Omega}$ and $\partial \Omega$, respectively, and suppose that the spectrum of $A_{1}(x)+i A_{2}(x)$ lies in the upper half-plane for all $x \in \bar{\Omega}$, and that $\operatorname{det}\left(B_{1}(y)+i B_{2}(y)\right) \neq 0$ for all $y \in \partial \Omega$. We call the following boundary value problem for the $\mathbb{C}^{r}$-valued function $w$,

$$
\begin{aligned}
& \frac{\partial w}{\partial x_{2}}-\left(A_{1}+i A_{2}\right) \frac{\partial w}{\partial x_{1}}=f(x), \quad x \in \Omega, \\
& \operatorname{Re}\left(B_{1}(y)-i B_{2}(y)\right) w=g(y), \quad y \in \partial \Omega,
\end{aligned}
$$

where $f=f_{1}+i f_{2}$ and $g$ is real-valued, a generalized Riemann-Hilbert problem.

Lemma 4.1. Consider the real $2 r \times 2 r$ boundary value problem for $v=\left[\begin{array}{ll}v_{1} & v_{2}\end{array}\right]^{T}$,

$$
\begin{gathered}
\frac{\partial v}{\partial x_{2}}-\left(\begin{array}{cc}
A_{1} & -A_{2} \\
A_{2} & A_{1}
\end{array}\right) \frac{\partial v}{\partial x_{1}}=\left(\begin{array}{l}
f_{1}(x) \\
f_{2}(x)
\end{array}\right), \quad x \in \Omega, \\
{\left[B_{1}(y) \quad B_{2}(y)\right] v=g(y), \quad y \in \partial \Omega .}
\end{gathered}
$$

There is a one-to-one correspondence between solutions of (2) and (1) given by $\left[\begin{array}{ll}v_{1} & v_{2}\end{array}\right]^{T} \mapsto w=v_{1}+i v_{2}$.

Proof. This is an easy consequence of the formula (3-4) and the fact that

$$
\frac{1}{2}\left(\begin{array}{c}
w \\
\bar{w}
\end{array}\right)=\left(\begin{array}{cc}
I & I \\
-i I & i I
\end{array}\right)^{-1}\left(\begin{array}{l}
v_{1} \\
v_{2}
\end{array}\right) .
$$

Left multiplication of the system (2) by $\left(\begin{array}{cc}I & I \\ -i I & i I\end{array}\right)^{-1}$ gives

$$
\frac{\partial}{\partial x_{2}}\left(\begin{array}{c}
w \\
\bar{w}
\end{array}\right)-\left(\begin{array}{cc}
A_{1}+i A_{2} & \\
& A_{1}-A_{2}
\end{array}\right) \frac{\partial}{\partial x_{1}}\left(\begin{array}{l}
w \\
\bar{w}
\end{array}\right)=\left(\begin{array}{l}
f(x) \\
\bar{f}(x)
\end{array}\right),
$$


which is just two copies of $\frac{\partial w}{\partial x_{2}}-\left(A_{1}+i A_{2}\right) \frac{\partial w}{\partial x_{1}}=f(x)$, and the boundary conditions in (2) may be written as

$$
\frac{1}{2}\left[B_{1}(y) \quad B_{2}(y)\right]\left(\begin{array}{cc}
I & I \\
-i I & i I
\end{array}\right)\left(\begin{array}{l}
w \\
\bar{w}
\end{array}\right)=g(y),
$$

that is, $\operatorname{Re}\left(B_{1}(y)-i B_{2}(y)\right) w=g(y)$. Conversely, if the function $w$ satisfies (1) then $v=\left[\begin{array}{ll}v_{1} & v_{2}\end{array}\right]^{T}$, where $v_{1}=(w+\bar{w}) / 2$ and $v_{2}=(w-\bar{w}) / 2 i$, satisfies $(2)$. This establishes the one-to-one correspondence between solutions of the two problems.

We call the boundary problem (2) the real form of the Riemann-Hilbert problem (1). Note that by Lemma 3.3 , we have

$$
\operatorname{det}\left(I_{2 r} \lambda-\left(\begin{array}{cc}
A_{1} & -A_{2} \\
A_{2} & A_{1}
\end{array}\right)\right)=\left|\operatorname{det}\left(I_{r} \lambda-\left(A_{1}+i A_{2}\right)\right)\right|^{2} \neq 0 \quad \text { for real } \lambda,
$$

so (2) is elliptic. Since $\operatorname{det}\left(B_{1}(y)-i B_{2}(y)\right) \neq 0$ for all $y \in \partial \Omega$, then it also satisfies the L-condition (Lemma 3.5). Hence the boundary problem (2) has a finite dimensional kernel and cokernel, and the same is therefore true of the RiemannHilbert problem (1). (We leave the verification of this fact to the reader.)

Let $(\mathbf{A}, \operatorname{Re}(B \cdot))$ denote the boundary problem (1), i.e. $\mathbf{A}=\partial / \partial x_{2}-$ $\left(A_{1}+i A_{2}\right) \partial / \partial x_{1}$ and $B=B_{1}-i B_{2}$. Now we construct a homotopy of elliptic operators A. Since the spectrum of $A_{1}+i A_{2}$ is contained in the upper half-plane, the same is true of $t\left(A_{1}+i A_{2}\right)+(1-t) i I$ when $0 \leq t \leq 1$. The operator

$$
\mathbf{A}^{(t)}=\partial / \partial x_{2}-\left(t\left(A_{1}+i A_{2}\right)+(1-t) i I\right) \partial / \partial x_{1}, \quad 0 \leq t \leq 1,
$$

is therefore elliptic, and the index, $\operatorname{ind}\left(\mathbf{A}^{(t)}, \operatorname{Re}(B \cdot)\right)$, is independent of $t$. With $t=1$ and $t=0$, it follows that

$$
\operatorname{ind}(\mathbf{A}, \operatorname{Re}(B \cdot))=\operatorname{ind}(-2 i \partial / \partial \bar{z}, \operatorname{Re}(B \cdot)) .
$$

Thus we have reduced the calculation of the index to the following boundary problem:

$$
\begin{gathered}
\frac{\partial w}{\partial \bar{z}}=h(z), \quad z=x_{1}+i x_{2} \in \Omega, \\
\operatorname{Re} B(y) w=g(y), \quad y \in \partial \Omega,
\end{gathered}
$$

where $B \in C^{\infty}(\partial \Omega, r \times r)$ and $\operatorname{det} B(y) \neq 0$ for all $y \in \partial \Omega$.

Theorem 4.2. The Riemann-Hilbert problem (3) has index

$$
\operatorname{ind}(\partial / \partial \bar{z}, \operatorname{Re}(B \cdot))=-\frac{1}{\pi}[\arg \operatorname{det} B(y)]_{\partial \Omega}+r .
$$

Proof. If the matrix $B(y)$ were diagonal, then (3) would be a diagonal system of $r$ scalar Riemann-Hilbert problems. Then (4) would follow directly from the index formula for the Riemann-Hilbert problem (see the introduction), by induction on the dimension $r$ of the system. However, the matrix function $B: \partial \Omega \rightarrow \mathrm{GL}_{r}(\mathbb{C})$ is homotopic to

$$
\left(\begin{array}{llll}
\operatorname{det} B(y) & & & \\
& 1 & & \\
& & \ddots & \\
& & & 1
\end{array}\right),
$$

so the theorem follows at once. 
Collecting all the preceding results, in particular, the system (2), Lemma 4.1 and Theorem 4.2, we obtain the index formula for first-order elliptic systems with real coefficients. Note that, by virtue of Lemma 2.6 , the quantity $\left[\arg \operatorname{det} B(y) X_{+}(y)\right]_{\partial \Omega}$ does not depend on the choice of $\gamma^{+}$-spectral pair $\left(X_{+}(x), T_{+}(x)\right), x \in \bar{\Omega}$.

Theorem 4.3. The index of the boundary value problem (1a), (1b) of $\S 3$ is

$$
\operatorname{ind}(\mathcal{A}, \mathcal{B})=-\frac{1}{\pi}\left[\arg \operatorname{det} B(y) X_{+}(y)\right]_{\partial \Omega}+r,
$$

where $X_{+}(x)$ is a $2 r \times r$ matrix function depending smoothly on $x \in \bar{\Omega}$ whose columns form a basis for the invariant subspace of $A(x)$ corresponding to the eigenvalues with positive imaginary part.

\section{INDEX FORMULAS FOR HIGHER-ORDER SYSTEMS WITH REAL COEFFICIENTS}

In this section we suppose that $\mathcal{A}$ is an elliptic operator with principal part homogeneous of degree $\ell$, and that the boundary operator $\mathcal{B}$ is a differential operator. In the first part of this section we also suppose that the principal part of $\mathcal{B}$ in each row is equal to the same number $\mu$; that is, with the notation of $\S 1,(\mathcal{A}, \mathcal{B}) \in \mathrm{BE}^{\ell, \mu}$. For the moment we also assume that $\mu \leq \ell-1$.

We will derive a formula for the index of $(\mathcal{A}, \mathcal{B})$ by reformulating $(\pi \mathcal{A}, \pi \mathcal{B})$ as a first-order system and then applying Theorem 4.3. In view of $\S 6$ we may suppose that $\mathcal{A}$ and $\mathcal{B}$ have matrix coefficients with real entries. Let

$$
L_{x}(\lambda)=\pi \mathcal{A}(x,(1, \lambda))=\sum_{j=0}^{\ell} A_{j}(x) \lambda^{j}, \quad x \in \bar{\Omega} .
$$

Since $\mathcal{A}$ is elliptic, then $A_{\ell}(x)=\pi \mathcal{A}(x,(0,1))$ is invertible for all $x \in \bar{\Omega}$. By considering the operator $A_{\ell}^{-1} \mathcal{A}$, we may assume that $A_{\ell} \equiv I$, that is, $L_{x}(\lambda)$ is monic. Also we let

$$
B_{y}(\lambda)=\pi \mathcal{B}(y,(1, \lambda))=\sum_{j=0}^{\ell-1} B_{j}(y) \lambda^{j}, \quad y \in \partial \Omega .
$$

Recall from $\S 1$ and Proposition 2.2 that $(\mathcal{A}, \mathcal{B})$ satisfies the L-condition if and only if $\operatorname{det} \Delta_{\mathcal{B}}^{+}(y) \neq 0$, where

$$
\Delta_{\mathcal{B}}^{+}(y)=\sum_{j=0}^{\ell-1} B_{j}(y) X_{+}(y) T_{+}^{j}(y) .
$$

As usual, we let $\left(X_{+}(x), T_{+}(x)\right)$ be a $\gamma^{+}$-spectral pair of $L_{x}(\lambda)$ consisting of smooth matrix functions on $\bar{\Omega}$. To reformulate $(\pi \mathcal{A}, \pi \mathcal{B})$ as a first-order system, let

$$
v_{j}=\frac{\partial^{\ell-1} u}{\partial x_{1}^{\ell-1-j} \partial x_{2}^{j}}, \quad j=0, \ldots, \ell-1,
$$

so that the equation $\pi \mathcal{A}(x, \partial / \partial x) u=f(x)$ takes the form

$$
\frac{\partial v_{\ell-1}}{\partial x_{2}}+A_{\ell-1}(x) \frac{\partial v_{\ell-1}}{\partial x_{1}}+\cdots+A_{0}(x) \frac{\partial v_{0}}{\partial x_{1}}=f(x),
$$

and there are the "compatibility conditions"

$$
\frac{\partial v_{j}}{\partial x_{2}}=\frac{\partial v_{j+1}}{\partial x_{1}}, \quad j=0, \ldots, \ell-2 .
$$


Thus, we obtain the first-order system

$$
\tilde{\mathcal{A}}(x, \partial / \partial x) v=\frac{\partial v}{\partial x_{2}}-C_{1}(x) \frac{\partial v}{\partial x_{1}}=F(x), \quad x \in \Omega .
$$

where $v=\left[\begin{array}{lll}v_{0} & \ldots & v_{\ell-1}\end{array}\right]^{T}$ and $F=\left[\begin{array}{llll}0 & \ldots & 0 & f\end{array}\right]^{T}$ are $p$-vector functions and $C_{1}(x)$ is the companion matrix for $L_{x}(\lambda)$ :

$$
C_{1}=\left(\begin{array}{ccccc}
0 & I & & & \\
0 & 0 & I & & \\
\vdots & \vdots & & \ddots & \\
0 & 0 & & I \\
-A_{0} & -A_{1} & & \ldots & -A_{\ell-1}
\end{array}\right) .
$$

Note that $\mathcal{A}$ is elliptic if and only if $\tilde{\mathcal{A}}$ is elliptic, since $\operatorname{det} L_{x}(\lambda)=\operatorname{det}\left(I \lambda-C_{1}(x)\right)$.

If $\mu=\ell-1$ the boundary conditions $\pi \mathcal{B}(y, \partial / \partial x) u=g(y)$ take the form

$$
\tilde{\mathcal{B}}(y) v=g(y), \quad y \in \partial \Omega,
$$

where $\tilde{\mathcal{B}}=\left[\begin{array}{llll}B_{0} & B_{1} & \ldots & B_{\ell-1}\end{array}\right]$, a $r \times p \ell$ matrix function. Then (1) can be written in the form

$$
\Delta_{\mathcal{B}}^{+}(y)=\tilde{\mathcal{B}} \cdot \operatorname{col}\left(X_{+}(y) T_{+}^{j}(y)\right)_{j=0}^{\ell-1} .
$$

Now we are ready to prove the first version of the index formula (for real coefficients). At first sight it might appear that (7) implies that the index of $(\mathcal{A}, \mathcal{B})$ depends only on the values of the coefficients of $\mathcal{A}$ on the boundary $\partial \Omega$, but in fact the choice of $\left(X_{+}, T_{+}\right)$, and hence $\Delta_{\mathcal{B}}^{+}$, depends on the coefficients throughout the region $\bar{\Omega}$.

Theorem 5.1. Let the hypotheses be as stated above: $(\mathcal{A}, \mathcal{B}) \in \mathrm{BE}^{\ell, \mu}$ (for arbitrary $\mu$ ) and the boundary value problem operators $\mathcal{A}$ and $\mathcal{B}$ have real matrix coefficients. Then

$$
\operatorname{ind}(\mathcal{A}, \mathcal{B})=-\frac{1}{\pi}\left[\arg \operatorname{det} \Delta_{\mathcal{B}}^{+}(y)\right]_{\partial \Omega}+r(2 \mu+2-\ell)
$$

Proof. Without loss of generality $(\mathcal{A}, \mathcal{B})=(\pi \mathcal{A}, \pi \mathcal{B})$, that is, $\mathcal{A}$ contains no derivatives of order $<\ell$ and $\mathcal{B}$ no derivatives of order $<\mu$. We prove the theorem in four steps: (1) $\mu=\ell-1$, and the operator $\mathcal{A}$ has constant coefficients; (2) $\mu=\ell-1$, for general $\mathcal{A}$; (3) $\mu<\ell-1$; and (4) $\mu \geq \ell$.

Step 1. We reformulate $(\mathcal{A}, \mathcal{B})$ as the first-order boundary value problem $(5 \mathrm{a})$, (5b) and apply Theorem 4.3 to $(\tilde{\mathcal{A}}, \tilde{\mathcal{B}})$. Note that the columns of

$$
\operatorname{col}\left(X_{+}(x) T_{+}^{j}(x)\right)_{j=0}^{\ell-1}
$$

form a basis for the invariant subspace of $C_{1}(x)$ corresponding to the eigenvalues with positive imaginary part. Thus by Theorem 4.3 and (6)

$$
\begin{aligned}
\operatorname{ind}(\tilde{\mathcal{A}}, \tilde{\mathcal{B}}) & =r-\frac{1}{\pi}\left[\arg \operatorname{det} \tilde{\mathcal{B}}(y) \cdot \operatorname{col}\left(X_{+}(x) T_{+}^{j}(x)\right)_{j=0}^{\ell-1}\right]_{\partial \Omega} \\
& =r-\frac{1}{\pi}\left[\arg \operatorname{det} \Delta_{\mathcal{B}}^{+}(y)\right]_{\partial \Omega} .
\end{aligned}
$$

We have the operators $(\mathcal{A}, \mathcal{B}): W_{2}^{\ell}\left(\Omega, \mathbb{R}^{p}\right) \rightarrow L_{2}\left(\Omega, \mathbb{R}^{p}\right) \times W_{2}^{1 / 2}\left(\partial \Omega, \mathbb{R}^{r}\right)$ and $(\tilde{\mathcal{A}}, \tilde{\mathcal{B}}):$ $W_{2}^{1}\left(\Omega, \mathbb{R}^{p \ell}\right) \rightarrow L_{2}\left(\Omega, \mathbb{R}^{p \ell}\right) \times W_{2}^{1 / 2}\left(\partial \Omega, \mathbb{R}^{r}\right)$, and to complete the proof of (7) we must 
show that

$$
\operatorname{ind}(\mathcal{A}, \mathcal{B})=\operatorname{ind}(\tilde{\mathcal{A}}, \tilde{\mathcal{B}})+r(\ell-1)
$$

There is a map $\Phi$ from $\operatorname{ker}(\mathcal{A}, \mathcal{B})$ to $\operatorname{ker}(\tilde{\mathcal{A}}, \tilde{\mathcal{B}})$ given by $u \mapsto v=\left[\begin{array}{lll}v_{0} & \cdots & v_{\ell-1}\end{array}\right]^{T}$, where $v_{j}$ are defined by $(2)$. Now, $\Phi$ is surjective for if $\tilde{\mathcal{A}} v=0$ and $\tilde{\mathcal{B}}(y) v=0$ then, by Weyl's lemma, $v \in C^{\infty}\left(\bar{\Omega}, \mathbb{R}^{p \ell}\right)$; since (4) holds and $\bar{\Omega}$ is contractible, then, by Lemma 5.2, there exists $u \in C^{\infty}\left(\bar{\Omega}, \mathbb{R}^{p}\right)$ such that (2) holds. Moreover, the kernel of $\Phi$ is the subspace $\mathcal{P}$ of all $\mathbb{R}^{p}$-valued polynomials $u$ of degree $\leq \ell-2$,

$$
u\left(x_{1}, x_{2}\right)=\sum_{j+k=0}^{\ell-2} c_{j k} x_{1}^{j} x_{2}^{k},
$$

where $c_{j k} \in \mathbb{R}^{p}$ are constants. Since the number of linearly independent monomials of the form $c_{j k} x_{1}^{j} x_{2}^{k}, j+k \leq \ell-2$, is equal to $1+2+\cdots+(\ell-1)=(\ell-1) \ell / 2$, it follows that

$$
\operatorname{dim} \mathcal{P}=p \cdot \frac{\ell(\ell-1)}{2}=r(\ell-1) .
$$

Hence $\operatorname{ker}(\mathcal{A}, \mathcal{B}) / \mathcal{P} \cong \operatorname{ker}(\tilde{\mathcal{A}}, \tilde{\mathcal{B}})$, so that

$$
\operatorname{dim} \operatorname{ker}(\mathcal{A}, \mathcal{B})=\operatorname{dim} \operatorname{ker}(\tilde{\mathcal{A}}, \tilde{\mathcal{B}})+r(\ell-1)
$$

At this point we wish to apply Lemma 5.3. The operator $\tilde{\mathcal{A}}: W_{2}^{\ell}\left(\Omega, \mathbb{R}^{p \ell}\right) \rightarrow$ $L_{2}\left(\Omega, \mathbb{R}^{p \ell}\right)$ is surjective for if $F \in C^{\infty}\left(\bar{\Omega}, \mathbb{R}^{p \ell}\right)$ we can extend $F$ to $C_{0}^{\infty}\left(\mathbb{R}^{2}, \mathbb{R}^{p \ell}\right)$ and then let $u=E * F$ where $E$ is a fundamental solution of $\tilde{\mathcal{A}}$; it follows that im $\tilde{\mathcal{A}} \supset$ $C^{\infty}\left(\bar{\Omega}, \mathbb{R}^{p \ell}\right)$. In virtue of Lemma 5.4 the image of $\tilde{\mathcal{A}}$ has finite codimensionbecause this is true of the image of $(\tilde{\mathcal{A}}, \tilde{\mathcal{B}})$-hence it must be closed. Hence $\tilde{\mathcal{A}}$ is surjective because we have shown that the image of $\tilde{\mathcal{A}}$ is closed and dense in $L_{2}\left(\Omega, \mathbb{R}^{p \ell}\right)$. Similarly $\mathcal{A}: W_{2}^{\ell}\left(\Omega, \mathbb{R}^{p}\right) \rightarrow L_{2}\left(\Omega, \mathbb{R}^{p}\right)$ is surjective. Indeed, this can be derived from the surjectivity of $\tilde{\mathcal{A}}$ for if $f \in C^{\infty}\left(\bar{\Omega}, \mathbb{R}^{p}\right)$ then there exists $v=$ $\left[\begin{array}{lll}v_{0} & \ldots & v_{\ell-1}\end{array}\right]^{T} \in C^{\infty}\left(\bar{\Omega}, \mathbb{R}^{p \ell}\right)$ such that $\tilde{\mathcal{A}} v=F=\left[\begin{array}{llll}0 & \ldots & 0 & f\end{array}\right]^{T}$, then $v$ satisfies (3), (4), and due to Lemma 5.2, there exists $u \in C^{\infty}\left(\bar{\Omega}, \mathbb{R}^{p}\right)$ such that (2) holds, whence $\mathcal{A} u=f$. Hence

$$
\begin{aligned}
\operatorname{ind}(\mathcal{A}, \mathcal{B}) & =\operatorname{ind}\left(\mathcal{B}_{\mid \operatorname{ker} \mathcal{A}}\right), \quad \text { by Lemma } 5.3 \\
& =\operatorname{ind}\left(\tilde{\mathcal{B}}_{\mid \operatorname{ker} \mathcal{A}_{1}}\right)+r(\ell-1), \quad \text { see }(10) \\
& =\operatorname{ind}(\tilde{\mathcal{A}}, \tilde{\mathcal{B}})+r(\ell-1), \quad \text { by Lemma } 5.3
\end{aligned}
$$

and then (8) implies

$$
\operatorname{ind}(\mathcal{A}, \mathcal{B})=-\frac{1}{\pi}\left[\arg \operatorname{det} \Delta_{\mathcal{B}}^{+}(y)\right]_{\partial \Omega}+r \ell,
$$

which is the formula (7) for the case $\mu=\ell-1$.

Step 2. It follows from the proof of Proposition 2.1 that $\bar{\Omega}$ is contractible, i.e. there exists a smooth map $\varphi: \bar{\Omega} \times I \rightarrow \bar{\Omega}$ such that $\varphi(x, 0)=x_{0}$ and $\varphi(x, 1)=x$ for all $x \in \bar{\Omega}$, where $x_{0}$ is a fixed point in $\bar{\Omega}$. Consider now the following homotopy of elliptic operators:

$$
\mathcal{A}_{t}(x, \partial / \partial x)=\mathcal{A}(\varphi(x, t), \partial / \partial x), \quad 0 \leq t \leq 1 .
$$

Note that $\mathcal{A}_{1}=\mathcal{A}$, and $\mathcal{A}_{0}$ has constant coefficients. In view of Proposition 1.2 there exists a homotopy $\left(\mathcal{A}_{t}, \mathcal{B}_{t}\right), 0 \leq t \leq 1$, of elliptic BVP's such that $\left(\mathcal{A}_{1}, \mathcal{B}_{1}\right)=(\mathcal{A}, \mathcal{B})$. 
Since the index is locally constant, we obtain $\operatorname{ind}(\mathcal{A}, \mathcal{B})=\operatorname{ind}\left(\mathcal{A}_{0}, \mathcal{B}_{0}\right)$. By Step 1 , we see that (11) holds for $\left(\mathcal{A}_{0}, \mathcal{B}_{0}\right)$. Thus it also holds for $(\mathcal{A}, \mathcal{B})$, since the winding number is locally constant and by Lemma 10.22 there exists a $\gamma^{+}$-spectral pair $\left(X_{+}, T_{+}\right)$for $L(\lambda)=\pi \mathcal{A}_{t}(x,(1, \lambda))$ which depends smoothly on $t$.

Step 3. Suppose now that $\mu<\ell-1$. We then operate on the boundary operator $\mathcal{B}$ with tangential derivatives to increase its order to $\ell-1$ :

$$
\mathcal{B}^{\prime}=(\partial / \partial \tau)^{\ell-1-\mu} \circ \mathcal{B},
$$

where $\partial / \partial \tau=\tau_{1} \partial / \partial x_{1}+\tau_{2} \partial / \partial x_{2}$. Corresponding to (1), let

$$
B_{y}^{\prime}(\lambda)=\pi \mathcal{B}^{\prime}(y,(1, \lambda))=\sum_{j=0}^{\ell-1} B_{j}^{\prime}(y) \lambda^{j}, \quad \Delta_{\mathcal{B}^{\prime}}^{+}(y)=\sum_{j=0}^{\ell-1} B_{j}^{\prime}(y) X_{+}(y) T_{+}^{j}(y) .
$$

Since

$$
B_{y}^{\prime}(\lambda)=\left(\tau_{1}(y)+\tau_{2}(y) \lambda\right)^{\ell-1-\mu} B_{y}(\lambda)
$$

then

$$
\Delta_{\mathcal{B}^{\prime}}^{+}(y)=\Delta_{\mathcal{B}}^{+}(y)\left(\tau_{1}(y) I+\tau_{2}(y) T_{+}(y)\right)^{\ell-1-\mu} .
$$

It follows that $\operatorname{det} \Delta_{\mathcal{B}^{\prime}}^{+}(y) \neq 0$ if and only if $\operatorname{det} \Delta_{+}(y) \neq 0$ since the spectrum, $\operatorname{sp}\left(T_{+}(y)\right)$, lies in the upper half-plane. Hence $\left(\mathcal{A}, \mathcal{B}^{\prime}\right)$ satisfies the L-condition. From (12) we see that

$$
\left[\arg \operatorname{det} \Delta_{\mathcal{B}^{\prime}}^{+}(y)\right]_{\partial \Omega}=\left[\arg \operatorname{det} \Delta_{\mathcal{B}}^{+}(y)\right]_{\partial \Omega}+(\ell-1-\mu)\left[\arg \operatorname{det}\left(\tau_{1} I+\tau_{2} T_{+}\right)\right]_{\partial \Omega}
$$

and, since $\frac{1}{2 \pi}\left[\arg \operatorname{det}\left(\tau_{1}(y) I+\tau_{2}(y) T_{+}(y)\right)\right]_{\partial \Omega}=r$, we obtain

$$
\frac{1}{\pi}\left[\arg \operatorname{det} \Delta_{\mathcal{B}^{\prime}}^{+}(y)\right]_{\partial \Omega}=\frac{1}{\pi}\left[\arg \operatorname{det} \Delta_{\mathcal{B}}^{+}(y)\right]_{\partial \Omega}+2 r(\ell-1-\mu) .
$$

Thus, to complete the proof of $(7)$, it suffices to show that $\operatorname{ind}\left(\mathcal{A}, \mathcal{B}^{\prime}\right)=\operatorname{ind}(\mathcal{A}, \mathcal{B})$. Consider the tangential derivative $\partial / \partial \tau$ as an operator from $W_{2}^{k+1}(\partial \Omega)$ to $W_{2}^{k}(\partial \Omega)$ for $k \geq 0$. This operator has index 0 , because the kernel of $\partial / \partial \tau$ is one-dimensional, consisting of the constant functions on $\partial \Omega$, and the image of $\partial / \partial \tau$ has codimension 1 , consisting of the functions $g \in W_{2}^{k}(\partial \Omega)$ such that $\int g=0$; therefore, ind $(\partial / \partial \tau)=$ $1-1=0$. It follows that the operator

$$
\Psi: W_{2}^{\ell}\left(\Omega, \mathbb{R}^{p}\right) \times W_{2}^{\ell-\mu-1 / 2}\left(\partial \Omega, \mathbb{R}^{r}\right) \longrightarrow W_{2}^{\ell}\left(\Omega, \mathbb{R}^{p}\right) \times W_{2}^{1 / 2}\left(\partial \Omega, \mathbb{R}^{r}\right)
$$

defined by $\Psi(f, g)=\left(f,(\partial / \partial \tau)^{\ell-1-\mu} g\right)$ is a composition of $\ell-1-\mu$ Fredholm operators, so $\Psi$ is also Fredholm, with index 0 . Then, since $\left(\mathcal{A}, \mathcal{B}^{\prime}\right)=\Psi \circ(\mathcal{A}, \mathcal{B})$, we obtain that $\operatorname{ind}\left(\mathcal{A}, \mathcal{B}^{\prime}\right)=\operatorname{ind} \Psi+\operatorname{ind}(\mathcal{A}, \mathcal{B})=\operatorname{ind}(\mathcal{A}, \mathcal{B})$. Consequently, by $(11)$ and (13)

$$
\begin{aligned}
\operatorname{ind}(\mathcal{A}, \mathcal{B}) & =\operatorname{ind}\left(\mathcal{A}, \mathcal{B}^{\prime}\right) \\
& =-\frac{1}{\pi}\left[\arg \operatorname{det} \Delta_{\mathcal{B}^{\prime}}^{+}(y)\right]_{\partial \Omega}+r \ell \\
& =-\frac{1}{\pi}\left[\arg \operatorname{det} \Delta_{\mathcal{B}}^{+}(y)\right]_{\partial \Omega}+r(2 \mu+2-\ell) .
\end{aligned}
$$

Step 4. Suppose that $\mu \geq \ell$. $(\mathcal{A}, \mathcal{B})$ is homotopic to $\left(\mathcal{A},(\partial / \partial \tau)^{\mu-\ell+1} \mathcal{R}\right)$, where $(\mathcal{A}, \mathcal{R}) \in \mathrm{BE}^{\ell, \ell-1}$ and $\tilde{\Delta}_{\mathcal{R}}^{+}=\tilde{\Delta}_{\mathcal{B}}^{+}$by Theorem 1.4. We then have

$$
\Delta_{\mathcal{R}}^{+}(y)=\Delta_{\mathcal{B}}^{+}(y) \cdot\left(\tau_{1}(y)+\tau_{2}(y) T_{+}(y)\right)^{\ell-1-\mu},
$$


for $y \in \partial \Omega$ (see Proposition 2.2). Hence

$$
\begin{aligned}
\operatorname{ind}(\mathcal{A}, \mathcal{B}) & =\operatorname{ind}(\mathcal{A}, \mathcal{R}) \\
& =-\frac{1}{\pi}\left[\arg \operatorname{det} \Delta_{\mathcal{R}}^{+}(y)\right]_{\partial \Omega}+r \ell \\
& =-\frac{1}{\pi}\left[\arg \operatorname{det} \Delta_{\mathcal{B}}^{+}(y)\right]_{\partial \Omega}-2 r(\ell-1-\mu)+r \ell, \quad \text { by }(14) \\
& =-\frac{1}{\pi}\left[\arg \operatorname{det} \Delta_{\mathcal{B}}^{+}(y)\right]_{\partial \Omega}+r(2 \mu+2-\ell),
\end{aligned}
$$

so that formula $(7)$ holds for $(\mathcal{A}, \mathcal{B})$. This completes the proof of the theorem.

Now we turn to the proof of the three lemmas which were used in the proof of Theorem 5.1.

Lemma 5.2. Let $v_{0}, \ldots, v_{\ell-1} \in C^{\infty}(\bar{\Omega}), \ell \geq 2$, such that

$$
\frac{\partial v_{j}}{\partial x_{2}}=\frac{\partial v_{j+1}}{\partial x_{1}}, \quad j=0, \ldots, \ell-2 .
$$

Then there exists $u \in C^{\infty}(\bar{\Omega})$ such that

$$
\frac{\partial^{\ell-1} u}{\partial x_{1}^{\ell-1-j} \partial x_{2}^{j}}=v_{j}, \quad j=0, \ldots, \ell-1 .
$$

Proof. Since $\bar{\Omega}$ is contractible then, by the Poincaré Lemma, every closed form on $\bar{\Omega}$ is exact. The lemma may be proved by induction on the number $\ell$.

Lemma 5.3. Let $E, E_{1}$ and $E_{2}$ be Banach spaces. Let $A: E \rightarrow E_{1}$ and $B$ : $E \rightarrow E_{2}$ be bounded linear operators. Let $A_{0}=A_{\mid \operatorname{ker} B}$. If $B$ is surjective then the following are equivalent:

(i) The operator $(A, B)$ from $E$ to $E_{1} \times E_{2}$ is Fredholm;

(ii) The operator $A_{0}$ from $\operatorname{ker} B$ to $E_{1}$ is Fredholm.

If $(A, B)$ and $A_{0}$ are Fredholm operators then $\operatorname{ind}(A, B)=\operatorname{ind} A_{0}$. (An analogous result is true if the roles of $A$ and $B$ are interchanged.)

Proof. The operators $(A, B)$ and $A_{0}$ have the same kernel. Also note that $f \in \operatorname{im} A_{0}$ if and only if $(f, 0) \in \operatorname{im}(A, B)$, so the map $f \mapsto(f, 0)$ induces a map

$$
E_{1} / \operatorname{im} A_{0} \longrightarrow\left(E_{1} \times E_{2}\right) / \operatorname{im}(A, B)
$$

which is injective. Hence

$$
\operatorname{codimim} A_{0} \leq \operatorname{codimim}(A, B) .
$$

If (i) holds then codim $\operatorname{im}(A, B)<\infty$ and thus codim im $A_{0}<\infty$ also. Since im $A_{0}$ has finite codimension it is closed and therefore (i) implies (ii). Conversely suppose (ii) holds. Let $(f, g) \notin \operatorname{im}(A, B)$. Since $B$ is surjective there exists $u \in E$ such that $B u=g$, then

$$
(f, g)=(f-A u, 0)+(A u, B u)
$$

which implies that the map (17) is surjective. Hence

$$
\operatorname{codimim}(A, B) \leq \operatorname{codimim} A_{0},
$$

so (ii) implies (i). In general we see that $\operatorname{im}(A, B)$ and $\operatorname{im} A_{0}$ have the same codimension, that is, either both dimensions are infinite or both are finite and equal. The last statement is now clear. 
We can also prove a more general result. Recall that for any Fredholm operator $A$, we denote the dimension of its kernel by $\alpha_{A}$ and the codimension of its image by $\beta_{A}$, and the index is defined by ind $A=\alpha_{A}-\beta_{A}$.

Lemma 5.4. Let $E, E_{1}$ and $E_{2}$ be Banach spaces. Let $A: E \rightarrow E_{1}$ and $B: E \rightarrow$ $E_{2}$ be bounded linear operators. Let $A_{0}=A_{\mid \operatorname{ker} B}$ and $B_{0}=B_{\mid \operatorname{ker} A}$. Then the following are equivalent:

(i) The image of the operator $\mathcal{L}=(A, B): E \rightarrow E_{1} \times E_{2}$ has finite codimension;

(ii) The images of the operator $A$ and of $B_{0}$ have finite codimension;

(iii) The images of the operator $A_{0}$ and of $B$ have finite codimension.

If any of (i), (ii), or (iii) hold then

$$
\beta_{\mathcal{L}}=\beta_{A}+\beta_{B_{0}}=\beta_{A_{0}}+\beta_{B}
$$

Since $\alpha_{\mathcal{L}}=\alpha_{A_{0}}=\alpha_{B_{0}}$ it follows that if any one of the operators $\mathcal{L}, A_{0}$ and $B_{0}$ is a Fredholm operator then all three are Fredholm and

$$
\text { ind } \mathcal{L}=\text { ind } B_{0}-\beta_{A}=\text { ind } A_{0}-\beta_{B} \text {. }
$$

Proof. There exist vector spaces $M_{0} \subset \operatorname{im} A$ and $N_{0} \subset \operatorname{im} B$ such that

$$
\operatorname{im} A=\operatorname{im} A_{0} \oplus M_{0}, \quad \operatorname{im} B=\operatorname{im} B_{0} \oplus N_{0}
$$

where $\oplus$ denotes a direct sum of vector spaces. Let

$$
\Gamma:=\left(M_{0} \times N_{0}\right) \cap \operatorname{im} \mathcal{L}
$$

We claim the following:

(a) $\Gamma$ is the graph of an invertible operator $M_{0} \rightarrow N_{0}$;

(b) $\operatorname{im} \mathcal{L}=\left(\operatorname{im} A_{0} \times \operatorname{im} B_{0}\right) \oplus \Gamma$;

(c) $\operatorname{im} A \times \operatorname{im} B=\operatorname{im} \mathcal{L} \oplus \hat{\Gamma}$, where $\hat{\Gamma}=\{(f,-g) ;(f, g) \in \Gamma\}$.

The proof is left as an exercise.

Now, to complete the proof of the lemma, let $k=\operatorname{dim} M_{0}=\operatorname{dim} N_{0}=\operatorname{dim} \Gamma=$ $\operatorname{dim} \hat{\Gamma}$. From (19) we have

$$
\beta_{A_{0}}=k+\beta_{A}, \quad \beta_{B_{0}}=k+\beta_{B} .
$$

Let $E_{1}=\operatorname{im} A \oplus M, E_{2}=\operatorname{im} B \oplus N$. In view of (c), we have

$$
E_{1} \times E_{2}=(\operatorname{im} A \times \operatorname{im} B) \oplus(M \times N)=\operatorname{im} \mathcal{L} \oplus \hat{\Gamma} \oplus(M \times N),
$$

whence

$$
\begin{aligned}
\beta_{\mathcal{L}} & =\operatorname{dim} \Gamma+\operatorname{dim} M+\operatorname{dim} N \\
& =k+\beta_{A}+\beta_{B},
\end{aligned}
$$

so the equalities in (18) hold. The equivalence of (i), (ii), and (iii) is now evident. The last statement in the lemma follows immediately.

When the orders of the rows of the boundary operator are not necessarily the same, Theorem 5.1 cannot be applied directly. However, the next theorem shows that the index can be written in terms of $\tilde{\Delta}_{\mathcal{B}}^{+}(y)=\sum_{j=0}^{\mu} \tilde{B}_{j}(y) \tilde{X}_{+}(y) \tilde{T}_{+}^{j}(y)$, where $\left(\tilde{X}_{+}(y), \tilde{T}_{+}(y)\right)$ is the $\gamma^{+}$-spectral pair defined by the transformation (2-2). In this form the index formula holds for any (differential) boundary operator $\mathcal{B}$. 
Theorem 5.5. Let $\mathcal{A} \in \mathrm{Ell}^{\ell}$ and let $\mathcal{B}$ be a differential boundary operator satisfying the $L$-condition (with no restrictions on the orders $m_{k}$ ). If $\mathcal{A}$ and $\mathcal{B}$ have real matrix coefficients then

$$
\operatorname{ind}(\mathcal{A}, \mathcal{B})=-\frac{1}{\pi}\left[\arg \operatorname{det} \tilde{\Delta}_{\mathcal{B}}^{+}(y)\right]_{\partial \Omega}+r(2-\ell)
$$

In other words, $\operatorname{ind}(\mathcal{A}, \mathcal{B})=\operatorname{ind}_{s}(\mathcal{A}, \mathcal{B})$ (see Definition 2.5$)$.

Proof. Let $m_{k}, k=1, \ldots, r$, denote the order of the $k$ th row of $\mathcal{B}$ and let $\mu=$ $\max _{k}\left(m_{k}\right)$. Now let

$$
\mathcal{B}^{\prime}(y, \partial / \partial x)=\left[\delta_{k j}(\partial / \partial \tau)^{\mu-m_{k}}\right]_{j, k=1}^{r} \circ \mathcal{B}(y, \partial / \partial x)
$$

so that $\mathcal{B}^{\prime}$ has the same order $\mu$ in each row. Then

$$
\pi \mathcal{B}^{\prime}(y, \xi)=\left[\delta_{k j}(\tau \cdot \xi)^{\mu-m_{k}}\right]_{j, k=1}^{r} \cdot \pi \mathcal{B}(y, \xi),
$$

thus $\pi \mathcal{B}^{\prime}(y, \tau+\lambda n) \equiv \pi \mathcal{B}(y, \tau+\lambda n)$. It follows that $\mathcal{B}^{\prime}$ satisfies the L-condition. Hence $\left(\mathcal{A}, \mathcal{B}^{\prime}\right) \in \mathrm{BE}^{\ell, \mu}$ and by Theorem 5.1

$$
\operatorname{ind}\left(\mathcal{A}, \mathcal{B}^{\prime}\right)=-\frac{1}{\pi}\left[\arg \operatorname{det} \Delta_{\mathcal{B}^{\prime}}^{+}(y)\right]_{\partial \Omega}+r(2 \mu+2-\ell)
$$

By Proposition 2.2 we also have

$$
\tilde{\Delta}_{\mathcal{B}^{\prime}}^{+}=\Delta_{\mathcal{B}^{\prime}}^{+} \cdot\left(\tau_{1} I+\tau_{2} T_{+}\right)^{-\mu}
$$

and, since $\frac{1}{2 \pi}\left[\arg \operatorname{det}\left(\tau_{1} I+\tau_{2} T_{+}\right)\right]_{\partial \Omega}=r$, it follows that

$$
-\frac{1}{2 \pi}\left[\arg \operatorname{det} \Delta_{\mathcal{B}^{\prime}}^{+}\right]_{\partial \Omega}=-\frac{1}{2 \pi}\left[\arg \operatorname{det} \Delta_{\mathcal{B}^{\prime}}^{+}\right]_{\partial \Omega}+\mu r .
$$

In view of (21) and (22), the formula (20) holds for the boundary value problem $\left(\mathcal{A}, \mathcal{B}^{\prime}\right)$. Since $\tilde{\Delta}_{\mathcal{B}^{\prime}}^{+}=\tilde{\Delta}_{\mathcal{B}}^{+}$and $\operatorname{ind}\left(\mathcal{A}, \mathcal{B}^{\prime}\right)=\operatorname{ind}(\mathcal{A}, \mathcal{B})$, it also holds for $(\mathcal{A}, \mathcal{B})$.

Example. Let $\mathcal{A}$ denote the elliptic operator defined in equation (19) of [Ro, §3], namely,

$$
\mathcal{A}\left(x, \frac{\partial}{\partial x}\right)=A(x) \frac{\partial^{2}}{\partial x_{2}^{2}}+2 B(x) \frac{\partial^{2}}{\partial x_{2} \partial x_{1}}+C(x) \frac{\partial^{2}}{\partial x_{1}^{2}}+\text { lower-order terms }
$$

where

$$
A(x)=\left(\begin{array}{cc}
\varphi_{1}(x) & \varphi_{2}(x)+1 \\
\varphi_{2}(x)-1 & -\varphi_{1}(x)
\end{array}\right), \quad B(x)=-I, \quad C(x)=A(x)^{T},
$$

and $\varphi_{1}, \varphi_{2}$ are real-valued functions in $C^{\infty}(\bar{\Omega})$ satisfying appropriate conditions. Consider once again the Dirichlet and Neumann problems for $\mathcal{A}$. Let $\left(\tilde{X}_{+}(y), \tilde{T}_{+}(y)\right)$ be the $\gamma^{+}$-spectral pair of $\tilde{L}_{y}(\lambda)$ defined by [Ro, (3-24), (3-25)]. For the Dirichlet problem $(\mathcal{A}, \mathcal{B})$ we showed that

$$
\operatorname{det} \Delta_{\mathcal{B}}^{+}(y, \tau(y))=i \overline{\varphi(y)}
$$

and hence it follows from Theorem 5.5 with $\ell=2$ that

$$
\begin{aligned}
\operatorname{ind}(\mathcal{A}, \mathcal{B}) & =-\frac{1}{\pi}\left[\arg \operatorname{det} \tilde{\Delta}_{\mathcal{B}}^{+}(y)\right]_{\partial \Omega} \\
& =\frac{1}{\pi}[\arg \phi(y)]_{\partial \Omega}
\end{aligned}
$$

Also, for the Neumann problem $(\mathcal{A}, \mathcal{C})$ we showed that

$$
\operatorname{det} \Delta_{\mathcal{C}}^{+}(y, \tau(y))=i \overline{\varphi(y)}\left(|\varphi(y)|^{2}-2\right)
$$


Since $|\varphi(y)|^{2}-2$ is real-valued it has zero winding number, and Theorem 5.5 implies

$$
\operatorname{ind}(\mathcal{A}, \mathcal{C})=\frac{1}{\pi}[\arg \phi(y)]_{\partial \Omega},
$$

the same as the index of the Dirichlet problem.

\section{Changing From Complex to Real matrix coefficients}

A polynomial with coefficients that are matrices with real entries shall be referred to as a real matrix polynomial. In other words, $L(\lambda)=\sum A_{j} \lambda^{j}$ is a real matrix polynomial if $\bar{A}_{j}=A_{j}$. Real matrix polynomials are also characterized by the following condition: $\overline{L(\lambda)}=L(\bar{\lambda})$ for all $\lambda \in \mathbb{C}$.

Throughout this section we assume that $\operatorname{det} L(\lambda) \neq 0$ for real $\lambda$. Also we let $\gamma^{ \pm}$be simple, closed contours containing the eigenvalues of $L(\lambda)$ in the upper and lower half-planes, respectively. Note that if $L(\lambda)$ is a real matrix polynomial and if $\left(X_{+}, T_{+}, Y_{+}\right)$is a $\gamma^{+}$-spectral triple of $L(\lambda)$, then $\left(\bar{X}_{+}, \bar{T}_{+}, \bar{Y}_{+}\right)$is a $\gamma^{-}$-spectral triple of $L(\lambda)$.

In general if $L(\lambda)=\sum A_{j} \lambda^{j}$ is a complex $p \times p$ matrix polynomial then $\overline{L(\lambda)}=$ $\bar{L}(\bar{\lambda})$ where $\bar{L}(\lambda):=\sum \bar{A}_{j} \lambda^{j}$ and $\bar{A}_{j}$ denotes the complex conjugate of $A_{j}$. Let $L_{1}(\lambda)$ and $L_{2}(\lambda)$ be the real and imaginary parts of $L(\lambda)$, i.e. the unique real $p \times p$ matrices such that $L(\lambda)=L_{1}(\lambda)+i L_{2}(\lambda)$. We then associate with $L(\lambda)$ the following real $2 p \times 2 p$ matrix polynomial:

$$
L_{\mathbb{R}}(\lambda)=\left(\begin{array}{cc}
L_{1}(\lambda) & -L_{2}(\lambda) \\
L_{2}(\lambda) & L_{1}(\lambda)
\end{array}\right)
$$

There is a close connection between the spectral data of $L(\lambda)$ and that of $L_{\mathbb{R}}(\lambda)$ (see Theorem 6.2). This can be anticipated by examining the differential equation $L(d / d t) u(t)=0$. If we write $u=u_{1}+i u_{2}$, then

$$
L_{\mathbb{R}}(d / d t) u_{\mathbb{R}}(t)=0, \quad \text { where } u_{\mathbb{R}}=\left(\begin{array}{l}
u_{1} \\
u_{2}
\end{array}\right) .
$$

For example, if $L\left(\lambda_{0}\right) x_{0}=0$ then the function $u(t)=e^{t \lambda_{0}} x_{0}$ is a solution of $L(d / d t) u=0$ and if we write $\lambda_{0}=a+i b$ and $x_{0}=x_{1}+i x_{2}$ then the corresponding real function

$$
\begin{aligned}
u_{\mathbb{R}}(t) & =\left(\begin{array}{c}
x_{1} \cos b t-x_{2} \sin b t \\
x_{1} \sin b t+x_{2} \cos b t
\end{array}\right) \\
& =\frac{1}{2} e^{t \lambda_{0}}\left(\begin{array}{c}
I \\
-i I
\end{array}\right) x_{0}+\frac{1}{2} e^{t \bar{\lambda}_{0}}\left(\begin{array}{c}
I \\
i I
\end{array}\right) \bar{x}_{0}
\end{aligned}
$$

satisfies $L_{\mathbb{R}}(d / d t) u_{\mathbb{R}}(t)=0$.

This implies $L_{\mathbb{R}}\left(\lambda_{0}\right) x_{0, \mathbb{R}}=0$ and $L_{\mathbb{R}}\left(\bar{\lambda}_{0}\right) \bar{x}_{0, \mathbb{R}}=0$, where $x_{0, \mathbb{R}}=\left(\begin{array}{c}I \\ -i I\end{array}\right) x_{0}$, which is easy to verify directly since

$$
L_{\mathbb{R}}\left(\lambda_{0}\right) x_{0, \mathbb{R}}=\left(\begin{array}{c}
L\left(\lambda_{0}\right) x_{0} \\
-i L\left(\lambda_{0}\right) x_{0}
\end{array}\right)=\left(\begin{array}{l}
0 \\
0
\end{array}\right) .
$$

Thus, to each eigenvalue $\lambda_{0}$ of $L(\lambda)$ with eigenvector $x_{0}$, there corresponds two eigenvalues, $\lambda_{0}$ and $\bar{\lambda}_{0}$, of $L_{\mathbb{R}}(\lambda)$ with eigenvectors $\left(\begin{array}{c}I \\ -i I\end{array}\right) x_{0}$ and $\left(\begin{array}{c}I \\ i I\end{array}\right) \bar{x}_{0}$, respectively. 
Lemma 6.1. Let $A$ and $B$ be $p \times p$ real matrices. Then

$$
\left(\begin{array}{cc}
A & -B \\
B & A
\end{array}\right)\left(\begin{array}{cc}
I & I \\
-i I & i I
\end{array}\right)=\left(\begin{array}{cc}
I & I \\
-i I & i I
\end{array}\right)\left(\begin{array}{cc}
A+i B & \\
& A-i B
\end{array}\right)
$$

and

$$
\operatorname{det}\left(\begin{array}{cc}
A & -B \\
B & A
\end{array}\right)=|\operatorname{det}(A+i B)|^{2}
$$

Proof. The first formula is proved by multiplying out both sides and comparing entries. The second formula follows immediately from the first.

By virtue of the lemma we have the following representation:

$$
L_{\mathbb{R}}(\lambda)=\left(\begin{array}{cc}
I & I \\
-i I & i I
\end{array}\right)\left(\begin{array}{ll}
L(\lambda) & \\
& \bar{L}(\lambda)
\end{array}\right)\left(\begin{array}{cc}
I & I \\
-i I & i I
\end{array}\right)^{-1}
$$

Since $\operatorname{det} L(\lambda) \neq 0$ for real $\lambda$ then $\operatorname{det} L_{\mathbb{R}}(\lambda) \neq 0$ for real $\lambda$. If $r$ (resp. $\alpha-r$ ) denotes the number of eigenvalues of $L(\lambda)$ in the upper (resp. lower) half-plane, counting multiplicities, then $L_{\mathbb{R}}(\lambda)$ has $r+(\alpha-r)=\alpha$ eigenvalues in the upper half-plane and also $\alpha$ eigenvalues in the lower half-plane.

Theorem 6.2. Let $L(\lambda)$ be a $p \times p$ matrix polynomial such that $\operatorname{det} L(\lambda) \neq 0$ for real $\lambda$. Let $\gamma^{ \pm}$be a simple, closed contour containing the eigenvalues of $L(\lambda)$ in the upper and lower half-planes, respectively. Let $\left(X_{ \pm}, T_{ \pm}, Y_{ \pm}\right)$be a $\gamma^{ \pm}$-spectral triple of $L(\lambda)$ consisting of matrices, i.e. $X_{+}$is a $p \times r$ matrix, $T_{+}$is $r \times r, Y_{+}$is $r \times p$, $X_{-}$is $p \times(\alpha-r), T_{-}$is $(\alpha-r) \times(\alpha-r)$ and $Y_{-}$is $(\alpha-r) \times p$. Then

$$
\begin{aligned}
X_{\mathbb{R}}^{+} & =\left(\begin{array}{cc}
I & I \\
-i I & i I
\end{array}\right)\left(\begin{array}{cc}
X_{+} & \\
& \bar{X}_{-}
\end{array}\right), \quad 2 p \times \alpha \text { matrix }, \\
T_{\mathbb{R}}^{+} & =\left(\begin{array}{ll}
T_{+} & \\
& \bar{T}_{-}
\end{array}\right), \quad \alpha \times \alpha \text { matrix, } \\
Y_{\mathbb{R}}^{+} & =\left(\begin{array}{ll}
Y_{+} & \\
& \bar{Y}_{-}
\end{array}\right)\left(\begin{array}{cc}
I & I \\
-i I & i I
\end{array}\right)^{-1}, \quad \alpha \times 2 p \text { matrix },
\end{aligned}
$$

is a $\gamma^{+}$-spectral triple of $L_{\mathbb{R}}(\lambda)$. (I denotes the $p \times p$ identity matrix.)

Proof. We shall verify (i), (ii'), (iii), and (iv) of the definition of spectral triples (see [Ro, Definition 2.1 and Proposition 2.2]).

(i) The fact that $\operatorname{sp}\left(T_{\mathbb{R}}^{+}\right)$lies inside $\gamma^{+}$is clear from the definition of $T_{\mathbb{R}}^{+}$.

(ii') Since $X_{ \pm} T_{ \pm}^{j} Y_{ \pm}=\frac{1}{2 \pi i} \int_{\gamma^{ \pm}} \lambda^{j} L^{-1}(\lambda)$ for $j=0,1, \ldots$ then

$$
\begin{aligned}
\bar{X}_{-} \bar{T}_{-}^{j} \bar{Y}_{-} & =\overline{\frac{1}{2 \pi i} \int_{\gamma^{-}} \lambda^{j} L^{-1}(\lambda) d \lambda} \\
& =-\frac{1}{2 \pi i} \int_{\gamma^{-}} \bar{\lambda}^{j} \bar{L}^{-1}(\bar{\lambda}) d \bar{\lambda} \\
& =\frac{1}{2 \pi i} \int_{\gamma^{+}} \lambda^{j} \bar{L}^{-1}(\lambda) d \lambda
\end{aligned}
$$

(Note that if $\gamma^{-}$is traversed in the counterclockwise sense, then $\overline{\gamma^{-}}$is traversed clockwise.) In view of (2) we have

$$
\int_{\gamma^{+}} \lambda^{j} L_{\mathbb{R}}^{-1}(\lambda) d \lambda=\left(\begin{array}{cc}
I & I \\
-i I & i I
\end{array}\right) \int_{\gamma^{+}} \lambda^{j}\left(\begin{array}{cc}
L^{-1}(\lambda) & \\
& \bar{L}^{-1}(\lambda)
\end{array}\right) d \lambda\left(\begin{array}{cc}
I & I \\
-i I & i I
\end{array}\right)^{-1}
$$


hence

$$
\begin{aligned}
\frac{1}{2 \pi i} \int_{\gamma^{+}} \lambda^{j} L_{\mathbb{R}}^{-1}(\lambda) d \lambda & =\left(\begin{array}{cc}
I & I \\
-i I & i I
\end{array}\right)\left(\begin{array}{ll}
X_{+} T_{+}^{j} Y_{+} & \\
& \bar{X}_{-} \bar{T}_{-}^{j} \bar{Y}_{-}
\end{array}\right)\left(\begin{array}{cc}
I & I \\
-i I & i I
\end{array}\right)^{-1} \\
& =X_{\mathbb{R}}^{+}\left(T_{\mathbb{R}}^{+}\right)^{j} Y_{\mathbb{R}}^{+}
\end{aligned}
$$

for $j=0,1, \ldots$.

(iii) From the definition of $X_{\mathbb{R}}^{+}$and $T_{\mathbb{R}}^{+}$, one obtains the following:

$$
\operatorname{col}\left(X_{\mathbb{R}}^{+}\left(T_{\mathbb{R}}^{+}\right)^{j}\right)_{j=0}^{\ell-1}=\left(\begin{array}{cc}
X_{+} & \bar{X}_{-} \\
-i X_{+} & i \bar{X}_{-} \\
X_{+} T_{+} & \bar{X}_{-} \bar{T}_{-} \\
-i X_{+} T_{+} & i \bar{X}_{-} \bar{T}_{-} \\
\vdots & \vdots \\
X_{+} T_{+}^{\ell-1} & \bar{X}_{-} \bar{T}_{-}^{\ell-1} \\
-i X_{+} T_{+}^{\ell-1} & i \bar{X}_{-} \bar{T}_{-}^{\ell-1}
\end{array}\right) .
$$

By means of block-row operations ( $i$ times the first block of $p$ rows, added to the second block of $p$ rows, etc.), the matrix (3) is transformed to

$$
\left(\begin{array}{cc}
X_{+} & \bar{X}_{-} \\
0 & 2 i \bar{X}_{-} \\
X_{+} T_{+} & \bar{X}_{-} \bar{T}_{-} \\
0 & 2 i \bar{X}_{-} \bar{T}_{-} \\
\vdots & \vdots \\
X_{+} T_{+}^{\ell-1} & \bar{X}_{-} \bar{T}_{-}^{\ell-1} \\
0 & 2 i \bar{X}_{-} \bar{T}_{-}^{\ell-1}
\end{array}\right)
$$

Since $\operatorname{col}\left(X_{+} T_{+}^{j}\right)_{j=0}^{\ell-1}$ and $\operatorname{col}\left(\bar{X}_{-} \bar{T}_{-}^{j}\right)_{j=0}^{\ell-1}$ have $r$ and $\alpha-r$ linearly independent columns, respectively, it is clear from the form of (4) that $\operatorname{col}\left(X_{\mathbb{R}}^{+}\left(T_{\mathbb{R}}^{+}\right)^{j}\right)_{j=0}^{\ell-1}$ has $\alpha$ linearly independent columns.

(iv) Similarly, one proves that $\operatorname{row}\left(Y_{\mathbb{R}}^{+}\left(T_{\mathbb{R}}^{+}\right)^{j}\right)_{j=0}^{\ell-1}$ has $\alpha$ linearly independent rows.

The following proposition will be of use in $\S 7$.

Proposition 6.3. Let the matrix polynomial $L(\lambda)$ and contours $\gamma^{+}$and $\gamma^{-}$be as in Theorem 6.2 , and $B(\lambda)=\sum_{j=0}^{\mu} B_{j} \lambda^{j}$ an $r \times p$ matrix polynomial of degree $\mu$. Let $\left(X_{ \pm}, T_{ \pm}\right)$be $\gamma^{ \pm}$-spectral pairs of $L(\lambda)$, respectively, and $\left(X_{\mathbb{R}}^{+}, T_{\mathbb{R}}^{+}\right)$the $\gamma^{+}$-spectral pair of $L_{\mathbb{R}}(\lambda)$ defined in Theorem 6.2. Also, let $B_{\mathbb{R}}(\lambda)=\sum_{j=0}^{\mu} B_{\mathbb{R}, j} \lambda^{j}$ be the real $2 r \times 2 p$ matrix polynomial corresponding to $B(\lambda)$. Then

$$
\Delta_{B_{\mathbb{R}}}^{+}=\left(\begin{array}{cc}
I_{r} & I_{r} \\
-i I_{r} & i I_{r}
\end{array}\right)\left(\begin{array}{cc}
\Delta_{B}^{+} & \\
& \overline{\Delta_{B}^{-}}
\end{array}\right)
$$

where $\Delta_{B_{\mathbb{R}}}^{+}=\sum_{j=0}^{\mu} B_{\mathbb{R}, j} X_{\mathbb{R}}^{+}\left(T_{\mathbb{R}}^{+}\right)^{j}$ and $\Delta_{B}^{ \pm}=\sum_{j=0}^{\mu} B_{j} X_{ \pm} T_{ \pm}^{j}$, and $I_{r}$ denotes the $r \times r$ identity matrix.

Proof. Write $B_{j}=M_{j}+i N_{j}$ where $M_{j}$ and $N_{j}$ are real $r \times p$ matrices. Then $B_{\mathbb{R}, j}=\left(\begin{array}{cc}M_{j} & -N_{j} \\ N_{j} & M_{j}\end{array}\right)$, so with the formula for $\left(X_{\mathbb{R}}^{+}, T_{\mathbb{R}}^{+}\right)$in Theorem 6.2 , it follows 
that

$$
\sum_{j=0}^{\mu} B_{\mathbb{R}, j} X_{\mathbb{R}}^{+}\left(T_{\mathbb{R}}^{+}\right)^{j}=\sum_{j=0}^{\mu}\left(\begin{array}{cc}
M_{j} & -N_{j} \\
N_{j} & M_{j}
\end{array}\right)\left(\begin{array}{cc}
I & I \\
-i I & i I
\end{array}\right)\left(\begin{array}{cc}
X_{+} T_{+}^{j} & \\
& \bar{X}_{-} \bar{T}_{-}^{j}
\end{array}\right)
$$

where $I$ denotes the $p \times p$ identity matrix. As in Lemma 6.1, it is easily verified that

$$
\left(\begin{array}{cc}
M_{j} & -N_{j} \\
N_{j} & M_{j}
\end{array}\right)\left(\begin{array}{cc}
I & I \\
-i I & i I
\end{array}\right)=\left(\begin{array}{cc}
I_{r} & I_{r} \\
-i I_{r} & i I_{r}
\end{array}\right)\left(\begin{array}{ll}
B_{j} & \\
& \bar{B}_{j}
\end{array}\right)
$$

and now (5) follows immediately.

Remark. Note that the matrix $\Delta_{B_{\mathbb{R}}}^{+}$is square $(2 r \times 2 r)$ exactly when $2 r$ is equal to the degree of $\operatorname{det} L(\lambda)$, which is the condition of proper ellipticity.

Let $\mathcal{A} \in$ Ell $^{\ell}$ be an elliptic operator in $\Omega \subset \mathbb{R}^{n}$ and $\mathcal{B}$ any differential boundary operator. If

$$
\mathcal{A}(x, \partial)=\sum A_{\alpha}(x) \partial^{\alpha}
$$

we let

$$
\overline{\mathcal{A}}(x, \partial)=\sum \bar{A}_{\alpha}(x) \partial^{\alpha}
$$

that is, the coefficients $A_{\alpha}$ are replaced by their complex conjugates $\bar{A}_{\alpha}$. Similarly for $\overline{\mathcal{B}}(y, \partial)$. Since $\mathcal{A} u=f, \mathcal{B} u=g$ if and only if $\overline{\mathcal{A}} \bar{u}=\bar{f}, \overline{\mathcal{B}} \bar{u}=\bar{g}$, there is a one-to-one correspondence between the solution spaces of $(\mathcal{A}, \mathcal{B})$ and $(\overline{\mathcal{A}}, \overline{\mathcal{B}})$; thus one problem satisfies the L-condition if and only if the other does, and $\operatorname{ind}(\overline{\mathcal{A}}, \overline{\mathcal{B}})=$ $\operatorname{ind}(\mathcal{A}, \mathcal{B})$.

If $\mathcal{A}=\mathcal{A}_{1}+i \mathcal{A}_{2}$, where the coefficients of $\mathcal{A}_{j}$ are real $p \times p$ matrix functions, we define

$$
\mathcal{A}_{\mathbb{R}}(x, \partial)=\left(\begin{array}{cc}
\mathcal{A}_{1}(x, \partial) & -\mathcal{A}_{2}(x, \partial) \\
\mathcal{A}_{2}(x, \partial) & \mathcal{A}_{1}(x, \partial)
\end{array}\right)
$$

which is a differential operator with coefficients that are real $2 p \times 2 p$ matrix functions. Similarly, if $\mathcal{B}=\mathcal{B}_{1}+i \mathcal{B}_{2}$, where the coefficients of $\mathcal{B}_{j}$ are real $r \times p$ matrix functions, we define

$$
\mathcal{B}_{\mathbb{R}}(y, \partial)=\left(\begin{array}{cc}
\mathcal{B}_{1}(y, \partial) & -\mathcal{B}_{2}(y, \partial) \\
\mathcal{B}_{2}(y, \partial) & \mathcal{B}_{1}(y, \partial)
\end{array}\right)
$$

is a differential operator with coefficients that are real $2 r \times 2 p$ matrix functions. Due to Lemma 6.1, there is a one-to-one correspondence between solutions of

$$
\begin{aligned}
& \mathcal{A}_{\mathbb{R}}(x, \partial)\left(\begin{array}{l}
u_{1} \\
u_{2}
\end{array}\right)=\left(\begin{array}{l}
f_{1}(x) \\
f_{2}(x)
\end{array}\right), \quad x \in \Omega, \\
& \mathcal{B}_{\mathbb{R}}(y, \partial)\left(\begin{array}{l}
u_{1} \\
u_{2}
\end{array}\right)=\left(\begin{array}{l}
g_{1}(y) \\
g_{2}(y)
\end{array}\right), \quad y \in \partial \Omega,
\end{aligned}
$$

and solutions of

$$
\begin{aligned}
&\left(\begin{array}{ll}
\mathcal{A}(x, \partial) & \\
\overline{\mathcal{A}}(x, \partial)
\end{array}\right)\left(\begin{array}{l}
v \\
\bar{v}
\end{array}\right)=\left(\begin{array}{l}
f(x) \\
\bar{f}(x)
\end{array}\right), x \in \Omega \\
&\left(\begin{array}{cc}
\mathcal{B}(y, \partial) & \overline{\mathcal{B}}(y, \partial)
\end{array}\right)\left(\begin{array}{l}
v \\
\bar{v}
\end{array}\right)=\left(\begin{array}{l}
g(y) \\
\bar{g}(y)
\end{array}\right), \quad y \in \partial \Omega
\end{aligned}
$$


where the correspondence is given by $v=u_{1}+i u_{2}, \bar{v}=u_{1}-i u_{2}$ (and $f=f_{1}+i f_{2}$, $\left.\bar{f}=f_{1}-i f_{2}\right)$. Without loss of generality $u_{1}$ and $u_{2}$ can be assumed to be real-valued in (6) (by taking real and imaginary parts). This does not affect the index.

Theorem 6.4. $\left(\mathcal{A}_{\mathbb{R}}, \mathcal{B}_{\mathbb{R}}\right)$ is L-elliptic if and only if $(\mathcal{A}, \mathcal{B})$ is L-elliptic. In that case, we have

$$
\operatorname{ind}\left(\mathcal{A}_{\mathbb{R}}, \mathcal{B}_{\mathbb{R}}\right)=2 \cdot \operatorname{ind}(\mathcal{A}, \mathcal{B})
$$

Proof. By Lemma 6.1

$$
\pi \mathcal{A}_{\mathbb{R}}(x, \xi)=\left(\begin{array}{cc}
I & I \\
-i I & i I
\end{array}\right)\left(\begin{array}{ll}
\pi \mathcal{A}(y, \xi) & \\
& \pi \overline{\mathcal{A}}(y, \xi)
\end{array}\right)\left(\begin{array}{cc}
I & I \\
-i I & i I
\end{array}\right)^{-1}
$$

for all $\xi \in \mathbb{R}^{n}$ (or $\mathbb{C}^{n}$ ). Hence $\operatorname{det} \pi \mathcal{A}_{\mathbb{R}}(x, \xi)=|\operatorname{det} \pi \mathcal{A}(x, \xi)|^{2}$ for all $\xi \in \mathbb{R}^{n}$; thus $\mathcal{A}_{\mathbb{R}}$ is elliptic if and only if the same is true of $\mathcal{A}$.

The $k$ th row of the boundary operator $\mathcal{B}_{\mathbb{R}}$ has order $\tilde{m}_{k}$ where

$$
\tilde{m}_{k}=m_{k}, \quad \tilde{m}_{k+r}=m_{k}, \quad k=1, \ldots, r,
$$

and we have

$$
\pi \mathcal{B}_{\mathbb{R}}(y, \xi)=\left(\begin{array}{cc}
I & I \\
-i I & i I
\end{array}\right)\left(\begin{array}{ll}
\pi \mathcal{B}(y, \xi) & \\
& \pi \overline{\mathcal{B}}(y, \xi)
\end{array}\right)\left(\begin{array}{cc}
I & I \\
-i I & i I
\end{array}\right)^{-1}
$$

The claim is that $\left(\mathcal{A}_{\mathbb{R}}, \mathcal{B}_{\mathbb{R}}\right)$ satisfies the L-condition if and only if the same is true of $(\mathcal{A}, \mathcal{B})$. Indeed, working in local admissible coordinates and replacing $\xi$ by $\left(\xi^{\prime}, \frac{1}{i} \frac{d}{d t}\right)$ in (8) and (9), we obtain that $W=\left(\begin{array}{l}w_{1} \\ w_{2}\end{array}\right)$ is a solution of the system

$$
\begin{gathered}
\pi \mathcal{A}_{\mathbb{R}}\left(y,\left(\xi^{\prime}, \frac{1}{i} \frac{d}{d t}\right)\right) W(t)=0, \quad t \geq 0, \\
\pi \mathcal{B}_{\mathbb{R}}\left(y,\left(\xi^{\prime}, \frac{1}{i} \frac{d}{d t}\right)\right) W(t)_{\mid t=0}=0,
\end{gathered}
$$

if and only if $w=w_{1}+i w_{2}, \bar{w}=w_{1}-i w_{2}$ is a solution of the system

$$
\begin{gathered}
\left(\begin{array}{r}
\pi \mathcal{A}\left(y,\left(\xi^{\prime}, \frac{1}{i} \frac{d}{d t}\right)\right) \\
\pi \overline{\mathcal{A}}\left(y,\left(\xi^{\prime}, \frac{1}{i} \frac{d}{d t}\right)\right)
\end{array}\right)\left(\begin{array}{l}
w(t) \\
\bar{w}(t)
\end{array}\right)=0, \quad t \geq 0, \\
\left(\begin{array}{rl}
\pi \mathcal{B}\left(y,\left(\xi^{\prime}, \frac{1}{i} \frac{d}{d t}\right)\right) & \overline{\mathcal{B}}\left(y,\left(\xi^{\prime}, \frac{1}{i} \frac{d}{d t}\right)\right)
\end{array}\right)\left(\begin{array}{l}
w(t) \\
\bar{w}(t)
\end{array}\right)_{\mid t=0}=0 .
\end{gathered}
$$

As pointed out earlier we may assume that $w_{1}$ and $w_{2}$ are real-valued. Then, by homogeneity of $\pi \mathcal{A}$, we have

Similarly,

$$
\pi \overline{\mathcal{A}}\left(y,\left(\xi^{\prime}, \frac{1}{i} \frac{d}{d t}\right)\right) \bar{w}(t)=(-1)^{\ell} \overline{\mathcal{A}\left(y,\left(-\xi^{\prime}, \frac{1}{i} \frac{d}{d t}\right)\right) w(t)} .
$$

$$
\pi \overline{\mathcal{B}}\left(y,\left(\xi^{\prime}, \frac{1}{i} \frac{d}{d t}\right)\right) \bar{w}(t)=M(-1) \overline{\pi \mathcal{B}\left(y,\left(-\xi^{\prime}, \frac{1}{i} \frac{d}{d t}\right)\right) w(t)} .
$$

It is now clear that (10) has the unique solution $W \equiv 0$ if and only if the system

$$
\begin{gathered}
\pi \mathcal{A}\left(y,\left(\xi^{\prime}, \frac{1}{i} \frac{d}{d t}\right)\right) w(t)=0, \quad t \geq 0, \\
\pi \mathcal{B}\left(y,\left(\xi^{\prime}, \frac{1}{i} \frac{d}{d t}\right)\right) w(t)_{\mid t=0}=0
\end{gathered}
$$


has the unique solution $w \equiv 0$. Moreover, due to the one-to-one correspondence between solutions of the boundary value problems (6) and (7), it follows that

$$
\begin{aligned}
\operatorname{ind}\left(\mathcal{A}_{\mathbb{R}}, \mathcal{B}_{\mathbb{R}}\right) & =\operatorname{ind}(\mathcal{A} \oplus \overline{\mathcal{A}}, \mathcal{B} \oplus \overline{\mathcal{B}}) \\
& =\operatorname{ind}(\mathcal{A}, \mathcal{B})+\operatorname{ind}(\overline{\mathcal{A}}, \overline{\mathcal{B}}) \\
& =2 \cdot \operatorname{ind}(\mathcal{A}, \mathcal{B}) .
\end{aligned}
$$

\section{The INDEX FORMUlA FOR ELLIPTIC SYSTEMS WITH COMPLEX COEFFiCIENTS} AND WHEN THE BOUNDARY OPERATOR IS PSEUDO-DIFFERENTIAL

First we extend Theorems 5.1 and 5.5 to the case when the boundary operator is differential with complex coefficients.

Theorem 7.1. If $(\mathcal{A}, \mathcal{B}) \in \mathrm{BE}^{\ell, \mu}$ then

(1) $\operatorname{ind}(\mathcal{A}, \mathcal{B})=-\frac{1}{2 \pi}\left[\arg \operatorname{det} \Delta_{\mathcal{B}}^{+}(y)\right]_{\partial \Omega}+\frac{1}{2 \pi}\left[\arg \operatorname{det} \Delta_{\mathcal{B}}^{-}(y)\right]_{\partial \Omega}+r(2 \mu+2-\ell)$.

Proof. Let $\left(\mathcal{A}_{\mathbb{R}}, \mathcal{B}_{\mathbb{R}}\right)$ and $L_{\mathbb{R}}(\lambda)$ denote the real boundary value problem operators and real matrix polynomial associated with $(\mathcal{A}, \mathcal{B})$ and $L(\lambda)$, respectively. By Theorem 6.2 , we have a $\gamma^{+}$-spectral pair $\left(X_{\mathbb{R}}^{+}(x), T_{\mathbb{R}}^{+}(x)\right)$ of $L_{\mathbb{R}, x}(\lambda)$ defined in terms of $\left(X_{ \pm}(x), T_{ \pm}(x)\right)$. Then, in virtue of Proposition 6.3,

$$
\Delta_{\mathcal{B}_{\mathbb{R}}}^{+}(y)=\left(\begin{array}{cc}
I_{r} & I_{r} \\
-i I_{r} & i I_{r}
\end{array}\right)\left(\begin{array}{cc}
\Delta_{\mathcal{B}}^{+}(y) & \\
& \overline{\Delta_{\mathcal{B}}^{-}(y)}
\end{array}\right)
$$

where $I_{r}$ denotes the $r \times r$ identity matrix. By Theorem 5.1,

$$
\operatorname{ind}\left(\mathcal{A}_{\mathbb{R}}, \mathcal{B}_{\mathbb{R}}\right)=-\frac{1}{\pi}\left[\arg \operatorname{det} \Delta_{\mathcal{B}_{\mathbb{R}}}^{+}(y)\right]_{\partial \Omega}+2 r(2 \mu+2-\ell),
$$

and, $\operatorname{since} \operatorname{ind}\left(\mathcal{A}_{\mathbb{R}}, \mathcal{B}_{\mathbb{R}}\right)=2 \cdot \operatorname{ind}(\mathcal{A}, \mathcal{B})$, the formula (1) follows immediately.

Remarks. (i) The formula (1) is not affected if the boundary operator $\mathcal{B}(y, \partial / \partial x)$ is replaced by $M(y) \mathcal{B}(y, \partial / \partial x)$, where $M(y)$ is an invertible $r \times r$ matrix function.

(ii) If the operators $\mathcal{A}$ and $\mathcal{B}$ have real matrix coefficients then the formula (1) is the same as that of Theorem 5.1 because we may let $\left(X_{-}, T_{-}\right)=\left(\bar{X}_{+}, \bar{T}_{+}\right)$ and therefore $\Delta_{\mathcal{B}}^{-}(y)=\overline{\Delta_{\mathcal{B}}^{+}(y)}$.

Theorem 7.2. If $\mathcal{A} \in \mathrm{Ell}^{\ell}$ and $\mathcal{B}$ is any differential boundary operator satisfying the L-condition (with no restrictions on the orders $m_{k}$ ) then

$$
\operatorname{ind}(\mathcal{A}, \mathcal{B})=-\frac{1}{2 \pi}\left[\arg \operatorname{det} \tilde{\Delta}_{\mathcal{B}}^{+}(y)\right]_{\partial \Omega}+\frac{1}{2 \pi}\left[\arg \operatorname{det} \tilde{\Delta}_{\mathcal{B}}^{-}(y)\right]_{\partial \Omega}+r(2-\ell) .
$$

Proof. This is derived from Theorem 5.5 in the same manner as above.

We now consider more general boundary operators. To do so we need to use Noether's formula for the index of p.d.o.'s on the unit circle in $\mathbb{R}^{2}$, and the formula of Agranovič-Dynin in [Ro, §6].

Denote by $\mathbf{T}$ the unit circle $|z|=1$ in $\mathbb{R}^{2}$. The volume element on $\mathbf{T}$ induced from $\mathbb{R}^{2}$ and the counterclockwise orientation is $\tau\left(e^{i x}\right)=x_{1} d x_{2}-x_{2} d x_{1}$, where $e^{i x}=x_{1}+i x_{2}$. If $\xi$ is a covector, we write $\xi>0$ if $\xi$ is a positive multiple of $\tau$, i.e. pointing in the counterclockwise direction. 
Theorem 7.3. Let $A \in \operatorname{OS}^{0}(\mathbf{T}, p \times p)$ with principal symbol $\pi A(z, \xi)=a_{+}(z)$, $\xi>0$, and $=a_{-}(z), \xi<0$, and suppose that $\operatorname{det} a_{ \pm}(z) \neq 0$ for all $z$. Then $A: L_{2}\left(\mathbf{T}, \mathbb{C}^{p}\right) \rightarrow L_{2}\left(\mathbf{T}, \mathbb{C}^{p}\right)$ is a Fredholm operator with index

$$
\text { ind } A=-\frac{1}{2 \pi}\left[\arg \operatorname{det} a_{+}\left(e^{i x}\right)\right]_{\mathbf{T}}+\frac{1}{2 \pi}\left[\arg \operatorname{det} a_{-}\left(e^{i x}\right)\right]_{\mathbf{T}} \text {. }
$$

This is a well-known result, due to Noether. For the proof, see [WRL, §8.10].

We now wish to consider general boundary operators, i.e. $\mathcal{B}=\sum_{j=0}^{\mu} \mathcal{B}_{j} D_{n}^{j}$ where the coefficients $\mathcal{B}_{j}$ are pseudo-differential operators on $\partial \Omega$. In its present form, the formula (2) does not hold for such boundary operators; however, if we define a $\gamma^{+}$spectral pair of $L_{y, \xi^{\prime}}(\lambda)$ as in $\S 1$ :

$$
\left(X_{+}\left(y, \xi^{\prime}\right), T_{+}\left(y, \xi^{\prime}\right)\right)=\left\{\begin{array}{cc}
\left(\tilde{X}_{+}(y), \tilde{T}_{+}(y)\right), & \xi^{\prime}=\tau(y), \\
\left(\tilde{X}_{-}(y),-\tilde{T}_{-}(y)\right), & \xi^{\prime}=-\tau(y),
\end{array}\right.
$$

then for a differential operator $\mathcal{B}$ we have

$$
\Delta_{\mathcal{B}}^{+}(y, \tau(y))=\tilde{\Delta}_{\mathcal{B}}^{+}(y) \quad \text { and } \quad \Delta_{\mathcal{B}}^{+}(y,-\tau(y))=M(-1) \tilde{\Delta}_{\mathcal{B}}^{-}(y) .
$$

Hence (2) can be written in the form (4) below, which turns out to be valid for any boundary operator.

Theorem 7.4. Let $\mathcal{A} \in \mathrm{Ell}^{\ell}$ and let $\mathcal{B}$ be any boundary operator satisfying the $L$-condition. Then with $\Delta_{\mathcal{B}}^{+}$defined with respect to the $\gamma^{+}$-spectral pair (3) we have

$$
\begin{aligned}
\operatorname{ind}(\mathcal{A}, \mathcal{B})= & -\frac{1}{2 \pi}\left[\arg \operatorname{det} \Delta_{\mathcal{B}}^{+}(y, \tau(y))\right]_{\partial \Omega} \\
& +\frac{1}{2 \pi}\left[\arg \operatorname{det} \Delta_{\mathcal{B}}^{+}(y,-\tau(y))\right]_{\partial \Omega}+r(2-\ell) .
\end{aligned}
$$

Proof. We have shown that $(4)$ holds for $\left(\mathcal{A}, \mathcal{B}^{\circ}\right)$ if $\mathcal{B}^{\circ}$ is a differential operator satisfying the $\mathrm{L}$-condition relative to $\mathcal{A}$ (for instance, let $\mathcal{B}^{\circ}$ be the boundary operator constructed in Theorem 2.4), that is,

$$
\begin{aligned}
\operatorname{ind}\left(\mathcal{A}, \mathcal{B}^{\circ}\right)= & -\frac{1}{2 \pi}\left[\arg \operatorname{det} \Delta_{\mathcal{B}^{\circ}}^{+}(y, \tau(y))\right]_{\partial \Omega} \\
& +\frac{1}{2 \pi}\left[\arg \operatorname{det} \Delta_{\mathcal{B}^{\circ}}^{+}(y,-\tau(y))\right]_{\partial \Omega}+r(2-\ell) .
\end{aligned}
$$

Now that we have the formula for one boundary problem, $\left(\mathcal{A}, \mathcal{B}^{\circ}\right)$, we can reduce the index problem for $(\mathcal{A}, \mathcal{B})$ to the calculation of an index on the boundary $\partial \Omega$, to which Noether's formula can be applied since $\partial \Omega$ is diffeomorphic to T. By [Ro, Theorem 6.2] we have

$$
\operatorname{ind}(\mathcal{A}, \mathcal{B})=\operatorname{ind}\left(\mathcal{A}, \mathcal{B}^{\circ}\right)+\operatorname{ind}\left(\Delta_{\mathcal{B}}^{+} \cdot\left(\Delta_{\mathcal{B}^{\circ}}^{+}\right)^{-1}\right)
$$

Since $\operatorname{ind}\left(\Delta_{\mathcal{B}}^{+} \cdot\left(\Delta_{\mathcal{B}^{\circ}}^{+}\right)^{-1}\right)=\operatorname{ind} \Delta_{\mathcal{B}}^{+}-\operatorname{ind} \Delta_{\mathcal{B}^{\circ}}^{+}$, then by applying Noether's formula to p.d.o.'s with principal symbols $\Delta_{\mathcal{B}}^{+}$and $\Delta_{\mathcal{B}^{\circ}}^{+}$on $\mathrm{ST}^{*}(\partial \Omega)$, respectively, (4) follows at once.

The next theorem gives a formula for the difference between the index of a given boundary value problem for $\mathcal{A}$ and the index of the Dirichlet problem. Here $\mathcal{A}$ is a properly elliptic operator of order $\ell=2 s$.

In order to state this result some notation is needed. For a boundary operator $\mathcal{B}$ we let

$$
G_{+}\left(y, \xi^{\prime}\right)=\frac{1}{2 \pi i} \int_{\gamma^{+}} B_{y, \xi^{\prime}}(\lambda) L_{y, \xi^{\prime}}^{-1}(\lambda)\left[\begin{array}{lll}
I & \ldots & \lambda^{s-1} I
\end{array}\right] d \lambda .
$$


In view of [Ro, Theorem 3.5], the boundary value problem $(\mathcal{A}, \mathcal{B})$ satisfies the Lcondition if and only if $\operatorname{det} G_{+}\left(y, \xi^{\prime}\right) \neq 0$ for all $y \in \partial \Omega, 0 \neq \xi^{\prime} \in T_{y}^{*}(\partial \Omega)$. The substitution $\lambda \rightarrow c^{-1} \lambda$ implies

$$
G_{+}\left(y, c \xi^{\prime}\right)=M(c) G_{+}\left(y, \xi^{\prime}\right) F(c) c^{1-\ell}, \quad \text { when } c>0,
$$

where $M(c)$ is defined as usual and $F(c)=\left[c^{j} \delta_{k j} I\right]_{k, j=0}^{s-1}$ is a $p s \times p s$ diagonal matrix. If $\mathcal{B}$ is a differential operator we also have (with $G_{-}$defined by the integral $\int_{\gamma^{-}}$)

$$
G_{+}\left(y,-\xi^{\prime}\right)=M(-1) G_{-}\left(y, \xi^{\prime}\right) F(-1)(-1)^{1-\ell} .
$$

For the Dirichlet problem the matrix functions $G_{ \pm}$take the form

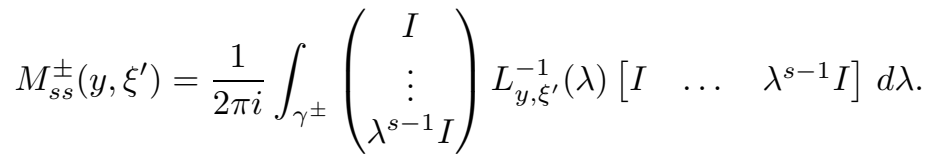

Note that $M_{s s}^{+}=-M_{s s}^{-}$because the integrand is $O\left(\lambda^{-2}\right)$ as $|\lambda| \rightarrow \infty$. (An application of Cauchy's theorem gives $\int_{\gamma^{+}}=\int_{-\infty}^{\infty}$ and $\int_{\gamma^{-}}=-\int_{-\infty}^{\infty}$. As usual, $\gamma^{+}$and $\gamma^{-}$are simple, closed contours in the upper and lower half-planes containing the eigenvalues of $L_{y, \xi^{\prime}}(\lambda)$ there, oriented in the counterclockwise direction.)

The following theorem is a particular case of the formula of Agranovič-Dynin, see [Ro, Theorem 6.2].

Theorem 7.5. Let $\mathcal{A} \in \mathrm{Ell}^{2 s}$ and let $\mathcal{B}$ be a boundary operator satisfying the $L$ condition. If the Dirichlet problem $(\mathcal{A}, \mathcal{D})$ satisfies the $L$-condition then

$$
\operatorname{ind}(\mathcal{A}, \mathcal{B})=\operatorname{ind}(\mathcal{A}, \mathcal{D})-\frac{1}{2 \pi}\left[\arg \operatorname{det} G_{+}(y, \tau(y))\right]_{\partial \Omega}+\frac{1}{2 \pi}\left[\arg \operatorname{det} G_{+}(y,-\tau(y))\right]_{\partial \Omega}
$$

Hence $\operatorname{ind}(\mathcal{A}, \mathcal{B})=\operatorname{ind}(\mathcal{A}, \mathcal{D})+\operatorname{ind} \mathcal{G}_{+}$, where $\mathcal{G}_{+}$is a p.d.o. on $\partial \Omega$ with principal symbol $G_{+}$.

Proof. Let $\left(X_{+}\left(y, \xi^{\prime}\right), T_{+}\left(y, \xi^{\prime}\right)\right)$ be the $\gamma^{+}$-spectral pair of $L_{y, \xi^{\prime}}(\lambda)$ defined as in (3). Let $\Delta_{+}=\Delta_{\mathcal{B}}^{+}$and $\Xi_{+}=\operatorname{col}\left(X_{+} T_{+}^{j}\right)_{j=0}^{s-1}$. By Theorem 7.4 we have

$$
\operatorname{ind}(\mathcal{A}, \mathcal{B})=-\frac{1}{2 \pi}\left[\arg \operatorname{det} \Delta_{+}(y, \tau(y))\right]_{\partial \Omega}+\frac{1}{2 \pi}\left[\arg \operatorname{det} \Delta_{+}(y,-\tau(y))\right]_{\partial \Omega}+r(2-\ell)
$$

and

$$
\operatorname{ind}(\mathcal{A}, \mathcal{D})=-\frac{1}{2 \pi}\left[\arg \operatorname{det} \Xi_{+}(y, \tau(y))\right]_{\partial \Omega}+\frac{1}{2 \pi}\left[\arg \operatorname{det} \Xi_{+}(y,-\tau(y))\right]_{\partial \Omega}+r(2-\ell) .
$$

Hence $\operatorname{ind}(\mathcal{A}, \mathcal{B})=\operatorname{ind}(\mathcal{A}, \mathcal{D})+\kappa$, where

$$
\kappa=-\frac{1}{2 \pi}[\arg \operatorname{det} R(y, \tau(y))]_{\partial \Omega}+\frac{1}{2 \pi}[\arg \operatorname{det} R(y,-\tau(y))]_{\partial \Omega}
$$

and $R=\Delta_{+} \cdot \Xi_{+}^{-1}$. Now, observe that

$$
\begin{aligned}
G_{+} & =\Delta_{+} \cdot\left[\begin{array}{lll}
Y_{+} & \ldots & T_{+}^{s-1} Y_{+}
\end{array}\right] \\
& =\Delta_{+} \Xi_{+}^{-1} \Xi_{+} \cdot\left[\begin{array}{lll}
Y_{+} & \ldots & T_{+}^{s-1} Y_{+}
\end{array}\right] \\
& =R \cdot M_{s s}^{+}
\end{aligned}
$$

As mentioned above, $M_{s s}^{+}=-M_{s s}^{-}$; thus (5) implies that $\operatorname{det} M_{s s}^{+}(y,-\tau(y))$ and $\operatorname{det} M_{s s}^{+}(y, \tau(y))$ are equal except for a constant factor \pm 1 . Hence they have the 
same winding number and

$$
\kappa=-\frac{1}{2 \pi}\left[\arg \operatorname{det} G_{+}(y, \tau(y))\right]_{\partial \Omega}+\frac{1}{2 \pi}\left[\arg \operatorname{det} G_{+}(y,-\tau(y))\right]_{\partial \Omega} .
$$

The last statement of the theorem now follows from Noether's formula for the index of p.d.o.'s on $\partial \Omega$ (Theorem 7.3).

Corollary 7.6. In addition to the hypotheses of Theorem 7.5, suppose that $\mathcal{B}$ is a differential operator and has order $\leq s-1$. Then

$$
\operatorname{ind}(\mathcal{A}, \mathcal{B})=\operatorname{ind}(\mathcal{A}, \mathcal{D}) \text {. }
$$

Proof. We define

$$
\tilde{G}_{ \pm}(y)=\frac{1}{2 \pi i} \int_{\gamma^{ \pm}} \tilde{B}_{y}(\lambda) \tilde{L}_{y}^{-1}(\lambda)\left[\begin{array}{lll}
I & \ldots & \lambda^{s-1} I
\end{array}\right] d \lambda
$$

and note that $G_{+}(y, \tau(y))=\tilde{G}_{+}(y)$. If $\mathcal{B}$ is a differential operator we also have

$$
G_{+}(y,-\tau(y))=M(-1) \cdot \tilde{G}_{-}(y) \cdot F(-1)(-1)^{1-\ell} .
$$

Thus Theorem 7.5 can also be stated as follows:

$$
\operatorname{ind}(\mathcal{A}, \mathcal{B})=\operatorname{ind}(\mathcal{A}, \mathcal{D})-\frac{1}{2 \pi}\left[\arg \operatorname{det} \tilde{G}_{+}(y)\right]_{\partial \Omega}+\frac{1}{2 \pi}\left[\arg \operatorname{det} \tilde{G}_{-}(y)\right]_{\partial \Omega} .
$$

Since the order of $\mathcal{B}$ is $\leq s-1$, the terms in the integrand of $\tilde{G}_{ \pm}(y)$ are $O\left(\lambda^{-2}\right)$ and we have

$$
\tilde{G}_{+}(y)-\tilde{G}_{-}(y)=\frac{1}{2 \pi i} \int_{\Gamma} \tilde{B}_{y}(\lambda) \tilde{L}_{y}^{-1}(\lambda)\left[\begin{array}{lll}
I & \ldots & \lambda^{s-1} I
\end{array}\right] d \lambda=0
$$

where $\Gamma$ is a simple closed contour containing all the roots of $\operatorname{det} \tilde{L}_{y}(\lambda)$ in its interior. Hence $\tilde{G}_{+}(y)=\tilde{G}_{-}(y)$ which implies $\operatorname{ind}(\mathcal{A}, \mathcal{B})=\operatorname{ind}(\mathcal{A}, \mathcal{D})$.

\section{REFERENCES}

[AB] M.F. Atiyah, R. Bott, The index problem for manifolds with boundary, Bombay Colloquium on Differential Analysis, Oxford Univ. Press, 1964, pp. 175-186. MR 32:3069

[AS] M.F. Atiyah, I.M. Singer, The index of elliptic operators I, Ann. of Math. 87 (1968), 484-530. MR 38:5243

[BGR] J.A. Ball, I. Gohberg, L. Rodman, Interpolation of Rational Matrix Functions, Operator Theory: Advances and Applications 45, Birkhäuser Verlag, Basel and Boston, 1990. MR 92m: 47027

[Ga] F.D. Gakhov, Boundary Value Problems, Dover Publications, New York, 1990. MR 45005

[GLR] I. Gohberg, P. Lancaster, L. Rodman, Matrix Polynomials, Academic Press, New York, 1982. MR 84c: 15012

[Ro] B. Rowley, Matrix polynomials and the index problem for elliptic systems, Trans. Amer. Math. Soc. 349 (1997), 3105-3148.

$[\mathrm{Ru}]$ W. Rudin, Real and Complex Analysis, Third Edition, McGraw-Hill, New York, 1987. MR 88k:00002

[Vo] A.I. Vol'pert, On the index and the normal solvability of boundary value problems for elliptic systems of differential equations in the plane, Trudy Mos. Mat. Obs. 10 (1961), 41-87 (Russian). MR 26:1603

[We] W.L. Wendland, Elliptic Systems in the Plane, Pitman, London, 1979. MR 89h:35053

[WRL] J. Wloka, B. Rowley, B. Lawruk, Boundary Value Problems for Elliptic Systems, Cambridge University Press, 1995. MR 96f:35003

Department of Mathematics, Champlain College, lennoxville, Quebec, Canada

E-mail address: browley@abacom.com 